Revised Version (MS \#1076) to Rheologica Acta, Dec. 16, 1999

\title{
Using Filament Stretching Rheometry to Predict Strand Formation and "Processability" in Adhesives and other Non-Newtonian Fluids
}

\author{
A. Tripathi ${ }^{1}$, P. Whittingstall ${ }^{2}$, G.H. McKinley ${ }^{1}$ \\ ${ }^{1}$ Dept. of Mechanical Engineering, M.I.T., Cambridge, MA 02139 \\ ${ }^{2}$ T.A. Instruments, New Castle, Delaware, DE 19720
}

\begin{abstract}
The spinning of polymeric fibers, the processing of numerous foodstuffs and the peel \& tack characteristics of adhesives is associated with the formation, stability and, ultimately, the longevity of thin fluid 'strands'. This tendency to form strands is usually described in terms of the tackiness of the fluid or by heuristic concepts such as 'stringiness' [Lakrout et al., J. Adhesion 69, 307 (1999)]. The dynamics of such processes are complicated due to spatially and temporally non-homogeneous growth of extensional stresses, the action of capillary forces and the evaporation of volatile solvents. We describe the development and application of a simple instrument referred to as a microfilament rheometer (MFR) that can be used to readily differentiate between the dynamical response of different pressure-sensitive adhesive fluid formulations. The device relies on a quantitative observation of the rate of extensional thinning or 'necking' of a thin viscous or viscoelastic fluid filament in which the solvent is free to evaporate across the free surface. This high-resolution measurement of the radial profile provides a direct indication of the ultimate time to break-up of the fluid filament. This critical time is a sensitive function of the rheological properties of the fluid and the mass transfer characteristics of the solvent, and can be conveniently reported in terms of a new dimensionless quantity we refer to as a Processability parameter $P$. We demonstrate the usefulness of this technique by presenting our results in the form of a case study in which we measure the visco-elasto-capillary thinning of slender liquid filaments for a number of different commercial polymer/solvent formulations and relate this to the reported processing performance of the materials. We also compare the MFR observations with the prediction of a simple 1D theory derived from the governing equations that model the capillary thinning of an adhesive filament.
\end{abstract}

Keywords: Capillary thinning, Filament stretching, Giesekus Model, Micro-filament Rheometer, Pressure Sensitive Adhesives, Processability, Spinnability, Stringiness. 


\section{Introduction}

Many important industrial processes involve the formation, elongation and ultimate breakage of thin liquid filaments or threads. Examples include the spinning of polymeric fibers (Ziabicki, 1976; Larson, 1983; Pearson, 1985), the processing of numerous foodstuffs (Padmanabhan, 1995) and the peel \& tack characteristics of adhesives (Gupta, 1991; Benyahia et al., 1997). For fluids exhibiting a complex rheological response such as polymer solutions \& melts, emulsions, suspensions of rigid rods or micellar fluids it is recognized that the rheological quantity of interest in understanding the dynamical characteristics of these processes is the extensional viscosity (Tanner, 1985). In particular, the transient extensional viscosity $\bar{\eta}^{+}\left(\dot{\varepsilon}_{0}, t\right)$ is a material function that characterizes the dynamical growth of the tensile stresses within the bulk of the fluid thread which resist the action of capillary forces and gravitational drainage attempting to break the filament.

For simple Newtonian fluids, Trouton (1906) was first to show that the extensional viscosity is simply a constant, equal in magnitude to three times the zero shear rate viscosity, such that $\bar{\eta}^{+}\left(\dot{\varepsilon}_{0}, t\right)=3 \eta_{0}$ (Tanner, 1985). Consequently no new material properties need to be measured, and the experimental and theoretical understanding of the visco-capillary break-up process in a number of different regimes is now well understood (see the recent review of Eggers, 1997). For non-Newtonian fluids with an underlying microstructure, however, constitutive modeling shows that the extensional viscosity is a nonlinear function of both the constant imposed elongation rate, $\dot{\varepsilon}_{0}$, and the elapsed time or, equivalently, the total elapsed Hencky strain $\varepsilon=\dot{\varepsilon}_{0} t$ of the deformation. Measurements of this material function can be performed in specially constructed devices (see for example Gupta \& Sridhar, 1988; James and Walters, 1993) and the resulting data can be used to extract values of the nonlinear model parameters that arise in the appropriate constitutive equation. The resulting model can then be used to predict the response of the non-Newtonian fluid in a more complex flow of processing interest. Connelly et al. (1983), Derail et al. (1997) and Piau et al. (1997) and, more recently, Christensen (1998) have shown how such measurements of the transient extensional viscosity in model pressure sensitive adhesives can be used to predict the peel force subsequently measured in a standard peeling experiment. 
In realistic commercial processes such as roll-coating of viscoelastic films, peel tests of adhesive strips, or the break-up of fluid filaments, the deformation experienced by a fluid element is neither spatially nor temporally homogeneous and hence the deformation rate $\dot{\varepsilon}(\boldsymbol{x}, t)$ becomes a function of position and time. To further complicate the issue, the presence or creation of a free surface may result in either mass transfer (e.g. the evaporation of a volatile solvent) or heat transfer (e.g. cooling of the fluid) which further change the rheological properties of the material being processed. These two physical phenomena are strongly coupled as a result of the highly nonlinear nature of the conservation equations describing the flow and deformation of the polymeric material being processed. For example, the evaporation of a volatile solvent from a polymer solution will result in an increase in the viscosity and relaxation time and thus modify critical processing characteristics such as the rate of thinning of a thread or leveling of a film. This change in the rate of creation (or elimination) of surface area can, in turn, further modify the mass transfer from the fluid sample. Several commercial examples of such processes are depicted schematically in figure 1.

The performance characteristics of such processes can be strongly dependent on the thickness uniformity of the final coating or related to the nature of instability that arise during the coating process. Several studies (Acrivos et al. (1960), Lawrence \& Zhou (1991), Britten \& Thomas (1992)) show how dramatically the non-Newtonian behavior of fluids (such as paints or photoresists) can lead to radially varying thicknesses in the final coatings. Recently, Eres et al. (1999) and Howison et al. (1997) presented mathematical models of a drying paint film on a two-dimensional, horizontal substrate using classical lubrication theory. On the experimental side, Birnie and Manley (1997) performed laser interferometry of solvent thinning behavior on spinning silicon wafers to yield plots of solvent thickness evolution, whilst the extensional and adhesion characteristics of a pressure sensitive adhesive have been measured by Ferguson et al. (1997) and Piau et al. (1997). Experiments described by Overdiep (1986) show that some solvent based high-gloss alkyd paints can exhibit more unusual behavior as they dry. Recent experiments by Kojima et al. (1995) show the same behavior in waterborne coatings with a high volatility co-solvent. Very few experimental studies have focused on quantifying the interaction of the non-Newtonian extensional viscosity and associated 
mass (or heat) transfer indicated in figure 1. However, all the aforementioned works support the general observation that liquid coating processes are rather complex and there is no simple theoretical or experimental way to predict the processability of adhesives or their tendency to exhibit flow instability (Coyle et al. (1990)).

In the present work we focus on one particular aspect of the final process depicted in figure 1(d); that is the formation and stability of thin fluid 'strands' during a process such as forward roll-coating of a liquid adhesive film. This tendency to form strands is poorly quantified at present and is usually described in terms of the tackiness of the fluid or by heuristic concepts such as 'stringiness' (Lakrout et al., 1999; Zosel, 1998; Wang et al., 1996; Taneya et al., 1992; Urahama, 1989). In the present paper, we describe a simple instrument referred to as a microfilament rheometer (MFR) that can be used to readily differentiate between the response of different adhesive fluid formulations. The device relies on a quantitative observation of the rate of extensional thinning of a viscoelastic fluid filament in which solvent is free to evaporate across the free surface. This measurement of the radial profile provides a direct indication of the ultimate time to break-up of the fluid filament. We demonstrate the technique's usefulness by presenting our results in the form of a case study in which we measure the visco-elasto-capillary thinning of slender liquid filaments for a number of different polymer/solvent formulations, We contrast these observations in our microfilament rheometer with conventional experimental studies that effectively isolate and measure individually either the mass transfer characteristics (e.g. using thermogravimetric analysis, TGA) or the nonlinear viscoelastic response (e.g. using steady shear rheometry). As we show, such data alone cannot successfully differentiate between the processing characteristics of the different fluids.

In the next section, we describe our experimental approach and show how simple filament stretching experiments can predict important processing features of adhesives. This leads to an explanation of our experimental technique and the results which were obtained for a series of adhesives. We then outline a simple 1D theory derived from the governing conservation equations for mass and momentum that models the visco-elastocapillary thinning of an adhesive filament. The final section is devoted to a brief discussion of the technique's applicability to other situations. 


\section{Experiments}

Table 1 lists 7 pressure sensitive adhesive formulations along with solvent compositions. The samples were all clear adhesives available commercially which were obtained by one of us (PW) for analysis. For convenience and confidentiality we refer to these as adhesive samples A1-A7. As a result of proprietary concerns the specific information regarding composition and solvent compatibility in these adhesives is not publishable. However such information can be obtained upon request from the authors. Such information is peripheral to our main objective of describing a new tool and method of analysis for understanding the "processability" of complex materials.

The seven adhesives used in this work were test-processed in an actual rollcoating device to investigate coating uniformity and effectiveness. It was found that the adhesives A5 \& A7 developed spatial instabilities (known as ribbing instabilities in the literature) instantaneously and it was not possible to use roll-coating devices for film production. These adhesives are referred to as exhibiting "bad" processability and this propensity to exhibit "stringing" is indicative of a high extensional viscosity. On the other hand, adhesives A2, A3 \& A6 were processed without any air bubbles or instability and thus exhibit "good" processability. Finally, it was found that certain adhesives such as A1 \& A4 could be processed for a period of time but eventually developed instabilities. These materials of intermediate properties to the two classes listed above were thus rated as exhibiting "ok" adhesives.

Since all samples contained volatile solvents, thermogravimetric measurements were performed first.

\subsection{Drying tests}

Samples of pressure sensitive adhesives consisted of a non-volatile resin/polymer dissolved in a volatile solvent. All samples exhibited evaporation of the volatile solvent. A thermogravimetric analyzer (DSC/TGA, Model 2970) was used to monitor the solvent loss as a function of time. All TGA experiments were performed at $24^{\circ} \mathrm{C}$. The drying was assumed to be isothermal and one-dimensional with negligible forced convection. Figure 2 shows a portion of the drying profiles of the adhesives. During the first 15 minutes of 
the thermogravimetric analysis, the drying process was extremely fast, and approximately $50 \%$ of the solvent evaporated. The sample denoted A6 dried fastest, whilst sample A5 exhibited the slowest rate of solvent loss. After about an hour, A6 and A7 had dried completely but samples such as A2 and A1 retained about $10 \%$ of the solvent.

The evaporation of a solvent from a pressure sensitive adhesive is governed by internal diffusion and by the interfacial mass transport between the adhesive and surrounding medium. If diffusion of solvent from the bulk of an adhesive to the surface is fast, then the process is mass transfer limited and the solvent concentration $c(t)$ in the adhesive can be estimated by

$$
\frac{d c}{d t}=-\frac{h_{m} A}{V} c-\frac{c}{V} \frac{d V}{d t}
$$

where $A$ and $V$ are the surface area and volume of the TGA sample, respectively, and $h_{m}$ is the mass transfer coefficient (with units of $\mathrm{m} / \mathrm{s}$ ) of the solvent at the sample surface. The third term $-c d \ln V / d t$ can be shown to be an order of magnitude smaller than the other two terms, hence it is ignored in further analysis. By integrating equation (1) it can be seen that the mass fraction of solvent decreases exponentially in time with a time constant $\sim\left(V / h_{m} A\right)$. The solid lines in figure 2 are the fits of an exponential decay in solvent concentration described by equation (1). A nonlinear regression method based on the Levenberg-Marquardt algorithm was used to extract the mass transfer coefficients, $h_{m}$, for the adhesives. For the sake of clarity, we only show three (A1, A2 \& A6) of the fitted exponential curves. The initial solvent mass fractions along with the mass transfer coefficients of all the adhesive samples are listed in Table 1. With the exception of A5 and A1, simple exponential curves fit the experimental data well. The maximum error in mass transfer coefficients was found to be about $3 \%$. The initial stages of the evaporation can be modeled even more accurately if the initial solvent concentration, $c_{0}$, is treated as an additional fitting parameter rather than a constant value as specified by supplier, however we have not explored this further in the present work.

\subsection{Rheological characterization and model fits}

A TA Instruments AR1000N controlled stress rheometer with normal force measurement capability was used to perform measurements of the viscoelastic properties 
of the materials in steady shear and small amplitude oscillatory shear flow. Since all samples showed a tendency to dry out in the rheometer, a $4 \mathrm{~cm}$ cone-and-plate fixture with an integral solvent trap and cover was used together with the appropriate solvent. Figures 3 and 4 present the steady shear viscosity, $\eta(\dot{\gamma})$, and first normal stress coefficient, $\Psi_{1}(\dot{\gamma})$, of all adhesive samples at $24^{\circ} \mathrm{C}$. Figure 3 shows that most of the adhesive samples exhibit shear thinning in the viscosity $\eta(\dot{\gamma})$ beyond applied shear rates of $10 \mathrm{~s}^{-1}$. The adhesives are viscoelastic and the first normal stress coefficient $\Psi_{1}(\dot{\gamma})$ shear thins monotonically throughout the entire range over which data can be obtained.

Nonlinear rheological parameters were determined by fitting the steady shear viscosity and first normal stress coefficient data to a single mode Giesekus model. All of the pressure sensitive adhesive formations considered here are concentrated polymer systems in organic solvents and we may expect a nonlinear network model such as the Giesekus model to predict an accurate description of the rheological properties (Yao et al. 1998, Li et al. 1998). We select the Giesekus model over other nonlinear differential constitutive equations such as the Phan-Thien-Tanner model since it contains only one nonlinear parameter which may be fully determined from shear flow experiments. Having determined all of the model parameters in linear viscoelastic and steady shear tests, it is then possible to use this model in a predictive capacity to see how well it describes a spatially and temporally nonhomogeneous flow such as elasto-capillary breakup.

In a single-mode formulation of this model, the solvent contribution $\tau_{s}$ and the polymeric contribution $\tau_{p}$ to the extra stress are defined as (Bird et.al, 1987)

$$
\begin{aligned}
& \tau=\tau_{s}+\tau_{p} \\
& \tau_{s}=\eta_{s}\left(\nabla \mathbf{v}+(\nabla \mathbf{v})^{T}\right) \\
& \tau_{p}+\lambda\left(\frac{\partial \tau_{p}}{\partial t}+\mathbf{v} \cdot \nabla \tau_{p}-(\nabla \mathbf{v})^{T} \cdot \tau_{p}-\tau_{p} \cdot \nabla \mathbf{v}\right)+\frac{\alpha \lambda}{\eta_{p}}\left\{\tau_{p} \cdot \tau_{p}\right\}=\eta_{p}\left(\nabla \mathbf{v}+(\nabla \mathbf{v})^{T}\right)
\end{aligned}
$$

where $\eta_{s}$ is the solvent contribution to viscosity. There are three additional independent physical parameters in equation (4): the polymer contribution to the viscosity, $\eta_{p}$; the polymer relaxation time $\lambda$; and the dimensionless mobility factor $\alpha$ associated with anisotropic effects. In the limit $\alpha \rightarrow 0$, the model reduces to the Oldroyd-B constitutive 
model. Equations (2)-(4) can be solved analytically for steady simple shear flow (see, e.g. Bird et. al (1987)) and the resulting predictions for the material functions $\eta(\dot{\gamma}), \Psi_{1}(\dot{\gamma})$ can be fitted to the experimental data by adjusting the values of $\alpha, \eta_{p}$ and $\lambda$ using an error minimization routine in the MATLAB numerical package. The solid lines in Figure 3 and 4 show model fits to the steady shear viscosity and normal stress coefficient data. For the sake of clarity, we have again shown representative fits for only three (A1, A2 and A6) of the adhesive formulations. The resulting parameter values for the single mode optimal fits of the single-mode Giesekus model to the experimental data are given in Table 2. Additional measurements of small amplitude oscillatory data were also found to be in fair agreement with the model fits, within the obvious inherent limitations of a single value for the relaxation time $\lambda$. Using a multiple relaxation time spectrum improves the description of the linear viscoelastic data but does not significantly improve the fit to the steady shear data. Furthermore the introduction of additional degrees of freedom increases the complexity of our analysis in $\$ 3$, without enhancing our understanding of the physical processes involved. We therefore proceed with a single mode analysis although this can be extended in a straightforward manner if so desired. Once the model parameters $\alpha, \eta_{p}$, and $\lambda$ are determined, the transient extensional rheological response predicted by the single mode Giesekus constitutive equations is fixed.

From the viscosity and normal force data alone it would seem that the four samples with the highest viscosity might well be the ones that process poorly, but the results from actual testing under coating conditions ranked the samples as seen in the Table 1. It is clear then that the "processability" of a sample cannot be predicted by steady and oscillatory shear flow data. Specifically, shear rheology fails to predict under what conditions samples were likely to perform well and which were likely to fail. Hence it is likely that some other parameter such as surface tension (interfacial energy) or elasticity might discriminate more effectively.

To express the relative importance of surface tension (listed in Table 2), the viscometric data can be converted to a dimensionless Capillary number, $C a=\eta \dot{\gamma} R / \sigma=\tau(\dot{\gamma}) R / \sigma$ and plotted as a function of shear rate (figure 5). Here, the characteristic length scale $R$ is taken to be the cone radius and $\sigma$ is the surface tension of 
the adhesive sample. Even though the surface tension is a function of solvent concentration, its dependence is rather weak compared to the dependence of viscosity on solvent concentration. The surface tension of these concentrated polymer/solvent systems is assumed to be a linear function of resin concentration (Ober et al. (1983)). The surface tension is then given by $\sigma=\sigma_{r}(1-x)+\sigma_{s} x$, where $\sigma_{r}$ and $\sigma_{s}$ are the surface tension of pure resin and pure solvent, respectively. Typical values of surface tension for pure organic solvents are 24 dynes/cm and for the pure polymeric resins are $30 \mathrm{dynes} / \mathrm{cm}$. Given the close proximity of these values, it is reasonable to approximate surface tension as a constant throughout the process. When expressed as a capillary number, the viscometeric data orders the samples in the same arrangement as the viscosity alone and it is clear that the processability of a sample can not be predicted in this way.

The relative importance of elastic effects in the adhesive liquids can be expressed in terms of a stress ratio or Weissenberg number $W i=N_{1}(\dot{\gamma}) / 2 \tau_{12}(\dot{\gamma})$. This dimensionless ratio of the first normal stress difference to the shear stress generated by the viscoelastic adhesive under steady shearing deformation is shown in figure 6. As can be seen in figure 6 the material responses are now separated into distinct curves and the samples that consistently fail in the processing tests have the highest Weissenberg numbers at a given shear rate indicating that the elasticity of the PSA is very important. However, since all of the curves increase monotonically with $\dot{\gamma}$, there is no way to estimate a dimensionless criterion such as a critical Weissenberg number $\mathrm{We}_{\text {crit }}$ beyond which processing of any chosen fluid formulation will become unstable.

In addition to governing the shear rheology of the test fluids, the values of the viscoelastic model parameters $\left\{\eta_{p}, \lambda, \alpha\right\}$ given in Table 2 also control the material response to extensional flows such as imposed stretching and localized necking (Yao et al. 1998; Hassager et al. 1998). In particular, for Weissenberg numbers $W i=\lambda \dot{\varepsilon}>0.5$ the steady state extensional viscosity is $\bar{\eta}(\dot{\varepsilon}) \cong 3 \eta_{s}+2 \eta_{p} / \alpha$ and is thus appreciably different for each fluid. Furthermore, theoretical studies of filament breakup (Renardy 1995, Entov and Hinch 1997) and numerical simulations (Bousfield et al. 1986, Yao et al. 1998) show that the rate of necking in a strongly strain-hardening material with $\alpha<<1$ is proportional to $(3 \lambda)^{-1}$. Hence, at this point we proceed to examine the hypothesis that 
extensional flow properties might be important in understanding the processability of adhesive materials.

\subsection{Filament Stretching experiments}

We analyze the capillary thinning of filaments of adhesive samples using a modified microfilament rheometer, sketched in figure 7. The original design for a Liquid Filament Rheometer (LFR) was discussed by Bazilevsky et al. (1990) and has been modified to allow convenient sample injection, control of the surrounding atmospheric environment and video imaging of the evolving filament profile. Liang and Mackley (1994) used a liquid filament rheometer to test several solutions of polyisobutylene in decalin and their data was further analyzed by Entov \& Hinch (1997). Recently, Kolte \& Szabo (1999) performed experiments in a new variant of the filament rheometer using a viscoelastic polymer solution with a non-volatile solvent and showed a good agreement with numerical simulations of the elastocapillary thinning process. To our knowledge, no studies investigating the combined effects of capillary thinning and mass transfer have been published.

The experimental operation of the MFR is rather simple. Initially, a cylindrical sample is loaded between two circular plates of radii $R_{0}=2.97 \mathrm{~mm}$ with initial plate separation $L_{0}=2.06 \mathrm{~mm}$ ( such that the initial aspect ratio $\Lambda_{0} \equiv L_{0} / R_{0}=0.693$ ). These plates are then rapidly separated $(\leq 0.1 \mathrm{~s})$ to a preset final displacement corresponding to $\Lambda=2.358$ by a spring mechanism. The rapid axial separation of the plates thus imparts a step extensional strain analogous to the step-shear strain experiment in a rheometer. The precise rate of separation and total time is not important provided that it is fast compared to any capillary break-up process. The sample is thereby stretched into an axisymmetric liquid bridge. The radial profile of the fluid column free surface, $R(z)$, is not axially uniform due to the no-slip boundary condition at either plate and is instead "necked" in the middle. Surface tension subsequently induces a thinning of the filament due to the non-uniform interfacial curvature, and, provided the filament is sufficiently long, it will break into two separate domains, one attached to each plate (Gillette and Dyson, 1971; Slobozhanin and Perales, 1993; Gaudet et al., 1996). The evolution in the mid-filament diameter can be measured using a CCD laser micrometer until very close to the ultimate 
breakup event. In our experiments, the global behavior of the liquid filament was also recorded on videotape. The video images were then analyzed on a computer to determine the mid-filament diameter and the axial profile.

Samples of the pressure sensitive adhesives were loaded through a small hole on the center of the top plate using a pressurized syringe system. During the loading procedure, the surrounding air space next to the plates of the MFR was saturated using the corresponding solvent in the sample. This ensures minimum evaporation of the solvent prior to the experimental measurement. Only one stretch was performed in each sample. Repeatability was verified using freshly loaded samples of each fluid formulation.

A representative series of images for two samples (A2 \& A5) undergoing elastocapillary thinning is shown in Figure 8. It is clear that the less viscous and less volatile sample A2 behaves entirely differently from sample A5 during the filament stretching process. While the A2 filament breaks in approximately $1.66 \mathrm{sec}$, the A5 filament does not break even after 20 minutes. The elasto-capillary breakup process for this material thus proceeds much more slowly than for the A2 filament. We also notice that the radial profiles of the necked regions of the filaments are almost symmetrical about the axial mid-plane. We can thus safely neglect the effect of gravity (as quantified by the dimensionless Bond number $B o=\rho g R_{0}^{2} / \sigma<1$ ) in the present study, except in the quasistatic regions near the upper and lower plates whose shapes are determined by a balance between gravity and capillarity. Figure 9 shows the transient midpoint diameter profiles for each fluid formulation measured in the micro-filament rheometer. It is clear that the samples A7 and A5 thin much more slowly compared to other samples. It is interesting to note that these profiles correspond directly to those fluids which were reported to exhibit bad processability by the suppliers (see Table 2). The samples A1 and A4 thin noticeably faster than A5 and A7 but slower than samples A6, A2 \& A3, and were denoted by the supplier as exhibiting "O.K." processability. Finally, adhesives A6, A2 and A3 undergo very rapid visco-elastic thinning and break into two within $1.6 \mathrm{sec}$. These materials are the ones rated by supplier as exhibiting "good" processability. The zero shear-rate viscosity of the adhesive A7 is seven times larger that of A2 or A3 and the typical capillary time scale $t_{\text {cap }} \sim \eta_{0} R_{0} / \sigma$ for evolution of the filament is thus increased 
accordingly. However, the breakup time for A7 is not seven times longer than A2 or A3. In fact, filaments of sample A7 never break. Although the shear viscosity may influence the prediction of "processability" (Chang, 1991), it is clear that a simple viscosity scaling alone fails in predicting actual breakup time of the filament.

It is apparent clear that the MFR technique can readily demarcate the adhesives in terms of their processability and this is reflected directly in the lifetime of thinning viscoelastic filaments. In order to understand the mechanism of this elastocapillary phenomenon in more detail, we present below a simple theoretical model capable of capturing the essential physics of visco-elasto-capillary thinning with combined mass transfer.

\section{Theoretical Predictions}

\subsection{Newtonian model}

Here we outline development of a simple 1D Newtonian model for predicting the thinning of adhesive fluid filaments due to capillary forces in the presence of a volatile solvent component.

Following Renardy (1995) and Yao et al. (1998), we consider the evolution of an axisymmetric viscoelastic filament constrained between circular plates. Since the filament is long and slender, it is assumed that fluid stresses and deformations can be regarded as uniform across any axial cross section. Furthermore, we neglect inertia and gravity. The tensile force acting on any cross section of the filament is then

$$
F=\pi R^{2}\left(T_{z z}-T_{r r}\right)+\pi R \sigma
$$

where $\sigma$ is the surface tension coefficient, $R(z, t)$ is the radius of the filament at axial position $z$ and time $t$, and $T_{z z}$ and $T_{r r}$ denote the (radially averaged) axial and radial components of the extra stress tensor resulting from fluid motion. Here higher order terms resulting from the axial curvature of the free surface have been neglected (i.e. we assume $\partial R / \partial z<<1$ ). Equation (5) can be re-formulated in a Lagrangian representation where initial fluid labels $Z_{0}$ (at time $t_{0}$ ) are followed as a function of time. The deformation in the fluid filament is represented by the dimensionless stretch $S\left(Z_{0}, t\right)$ of

each element which is defined in a 1D deformation by 
$\left(\partial z / \partial Z_{0}\right)_{t} \equiv S\left(Z_{0}, t\right)=R_{0}^{2} / R(z, t)^{2}$ (Renardy, 1995). The deformation rate within the filament is given by

$$
\dot{\varepsilon}(z, t)=\frac{1}{S} \frac{\partial S}{\partial t}=-\frac{2}{R} \frac{\partial R}{\partial t}
$$

Substituting for the Newtonian constitutive relationship $\left(T_{z z}-T_{r r}\right)=3 \eta_{s} \dot{\varepsilon}$ in equation (5) and non-dimensionalizing leads to

$$
f(t)=3 \eta_{r}(t) \frac{1}{S^{2}} \frac{\partial S}{\partial t}+\frac{1}{\sqrt{S}}
$$

where $f(t)=F / \sigma \pi R_{0}$ is the scaled force, and $\eta_{r}=\eta(t) / \eta_{0}\left(t_{0}\right)$ is the relative viscosity of the adhesive at time $t$ to the initial viscosity at $t_{0}$. Here, time is nondimensionlized with the characteristic capillary time scale $t_{c a p}=\eta_{0} R_{0} / \sigma$. Since the end plates are not moving and the global or total stretch of the entire filament is constant, the force can be found from an integral constraint (Renardy, 1995) as

$$
f(t)=\frac{\int_{0}^{\Lambda_{0}} S^{3 / 2} / \eta_{r} d Z_{0}}{\int_{0}^{\Lambda_{0}} S^{2} / \eta_{r} d Z_{0}}
$$

where the relative viscosity is a function of time as well as axial position.

It remains to estimate the variation in the viscosity of the adhesive liquid due to evaporation effects by using mass transfer principles. We expect that the extensional viscosity of the polymeric fluid and the increase in the viscoelastic properties of the fluid due to solvent evaporation both affect the overall rate of capillary thinning during the necking of the fluid filament. The coating materials studied in this work are modeled as simple binary liquids composed of "resin" or polymer and a volatile "solvent." Only the solvent evaporates and, as it does, the concentration of polymer in the bulk adhesive increases. As a result of the axial slenderness of the filament, we assume that the solvent diffusion process within the bulk of polymer is much faster than its evaporation from the interface and radial gradients in the concentration can be neglected so that $c(\boldsymbol{x}, t) \rightarrow c(z, t)$. This approximation will become increasingly good as the necking progresses and the filament becomes increasingly slender, since the surface/volume ratio increases monotonically. The solvent concentration $c$ in any axial slice of an adhesive 
filament is thus governed by equation (1). The mass balance for each Lagrangian element $Z_{0}$ can be written as

$$
\left(\frac{\partial c}{\partial t}\right)_{Z_{0}}=-\frac{2 h_{m} c(z, t)}{R(z, t)}
$$

Finally, for algebraic simplicity, we assume that the density of polymer and the solvent are equal at all times so that the density of the adhesive fluid remains unchanged during the evaporation. Non-dimensionalizing equation (9) using the (constant) density, the stretch $S$ and the characteristic time scale $t_{c a p}=\eta_{0} R_{0} / \sigma$ leads to

$$
\left(\frac{\partial x}{\partial t}\right)_{Z_{0}}=-\left(\frac{2 h_{m} \eta_{0}}{\sigma}\right) x \sqrt{S}
$$

where $x(z, t)=c / \rho$ is the mass fraction of solvent in the filament at each axial slice. The important point to note from equation (10) is that as the filament of adhesive fluid thins under capillary pressure, the interfacial area and axial stretch increase and thus the rate of mass transfer across the surface also increases. As we show below, this positive feedback mechanism leads to very rapid formation of thin strands and is manifested in processing operations as "stringiness".

The dimensionless group characterizing the rate of increase in mass transfer in equation (10) may be interpreted as a ratio of the two time scales in the problem. The characteristic time scale for solvent evaporation is $t_{\text {evap }} \sim R_{0} / h_{m}$ and the time scale for capillary thinning is $t_{c a p} \sim \eta_{0} R_{0} / \sigma$ (for a Newtonian filament). We have not been able to identify this dimensionless group in the existing literature and thus define it a processability parameter $P$. Note, however, that the parameter $P$ can be written in terms of other well-known dimensionless groups as

$$
\frac{h_{m} \eta_{0}}{\sigma} \equiv P=\frac{S h C a}{\operatorname{Re} S c}=S t_{m} C a
$$

where $S h=h_{m} R_{0} / D$ is the Sherwood number with D the mass diffusivity of the solvent, $C a=\eta_{0} R_{0} \dot{\varepsilon}_{0} / \sigma$ is the capillary number, $R e=\rho \dot{\varepsilon}_{0} R_{0}^{2} / \eta_{0}$ is the Reynolds number and $S c=\eta_{0} / \rho D$ is the Schmidt number. The grouping $S h / R e S c$ is sometimes identified as the mass transfer Stanton number (Bird et al.,1960). Table 3 lists the processability parameter $P$ for all the samples. It is clear that the samples A7, A5, A1 and A4 all have 
the largest values of this processability parameter $P$. Furthermore these were the samples which exhibited the slowest rates of capillary thinning and also exhibiting instabilities during roll-coating. Hence, the magnitude of the parameter $P$ can be assessed as a measure of "stringiness" or "processability". Also listed in Table 3 are values of the elastocapillary number $\Gamma=\mathrm{De} / \mathrm{Ca}$ measuring the relative importance of viscoelasticity to surface tension which we discuss further in $\$ 3.2$ below.

Although we have not directly measured the variation of adhesive viscosity with solvent concentration, we relate the solvent composition to the bulk viscosity using a simple mixture rule of the form (Irving, 1977, Gambill, 1959)

$$
\frac{1}{\eta}=\frac{1-x}{\eta_{1}}+\frac{x}{\eta_{s}}
$$

where $\eta_{1}$ and $\eta_{s}$ are the zero-shear rate viscosities of the pure polymer and pure solvent respectively. We note parenthetically that alternate expressions for viscosity have been proposed by several authors (Irving, 1977), but the results to be presented below are not significantly affected if one expression is chosen over the other and the simple from of eq.(12) permits some analytical insight into the physical processes as we show below.

Combining equations (10) and (12) allows us to find the variation in viscosity with time during the capillary thinning process. If $\eta_{s} / \eta_{1}<<1$, and the mass fraction of solvent monotonically decreases with time (i.e. $0 \leq x(t) \leq x_{0}$ ) then equations (10) and (12) can be combined and simplified to give

$$
\eta_{r}=\frac{\eta_{0}(t)}{\eta_{0}\left(t_{0}\right)}=\exp \left\{2 P \int_{0}^{t} S^{\frac{1}{2}} d t\right\}
$$

Clearly incorporating the mass transfer of the solvent $(P \neq 0)$ results in the viscosity of the adhesive fluid increasing exponentially with the degree of stretch in the filament.

Given an initial filament profile $R\left(Z_{0}, t_{0}\right)$, Eqs. (7), (8) and (13) can be integrated in time to compute the evolution in the filament profile. If the filament breaks in finite time, then the Lagrangian stretch of the adhesive material element at the mid-point diverges at a critical time, $t_{c}$. In the microfilament rheometer first developed by Entov and coworkers (Bazilevsky et al., 1990), the initial profile of the filament at time $t_{0}$ is a cylindrical liquid bridge of aspect ratio $\Lambda_{0}=L_{0} / R_{0}$ which is rapidly deformed in a short 
time $\delta t$ to yield an initial "necked" filament profile $R_{1}\left(Z_{0}\right)$ and aspect ratio $\Lambda_{1}=L_{1} / R_{0}$ at time $t_{1}=t_{0}+\delta t$. This liquid bridge subsequently evolves due to capillarity.

This "necked" profile is the initial condition required for integrating equations (7), (8) and (13) and can be obtained from digitized video images of the initial filament profile $R_{1}\left(Z_{0}, t_{1}\right)$. Alternatively, for analytic simplicity, we generate initial conditions using the lubrication solution for reverse squeeze flow provided by Spiegelberg et al. (1996) for a given initial stretching of a viscous fluid sample from height $L_{0}$ to $L_{1}$. The partial differential equations are converted to a set of ordinary differential equations (ODE's) for a finite number of initial particle labels $Z_{0}^{[i]}, i=1 \ldots N$ and the filament profiles for different values of the processability parameter $P$ are obtained using a $4^{\text {th }}$ order Runge-Kutta integrator for stiff ODE's available in MATLAB. In the results shown here we use $N=201$ points to describe the initial necked profile $R_{1}\left(Z_{0}, t_{1}\right)$. We estimate values of the processability parameter $P$ for each of the adhesive samples from the mass transfer coefficients in Table 1, the initial viscosity $\eta_{0}$ in Table 2 and the surface tension, $\sigma$. These values are given in Table 3 .

In figure 10 we show representative transient profiles of the mid plane radius $R_{\text {mid }}(t)$ obtained numerically from equations (7), (8) and (13) for different Newtonian fluid formulations for parameters $P=0,0.005,0.01$ and 0.05 . These predictions show a remarkable similarity with the experimentally observed profiles presented in figure 9. It appears clear that the combination of effects resulting from evaporation of the volatile solvent component and visco-capillary thinning contributes to the experimentally observed "stranding" or "stringiness" of adhesive filaments and directly correlates the prediction of adhesive processability.

It is also possible to obtain a closed form solution for combined evaporation and Newtonian capillary thinning by assuming the filament shapes shown in figure 8 can be approximated as axially uniform cylindrical threads held between large quasi-static or stagnant drops (or "blobs") and then following the analysis of Entov \& Hinch (1997). The dotted lines in Figure 10 show the results of this analytical solution which is derived in the appendix. 


\subsection{Giesekus model for viscoelastic-capillary drainage of adhesive filaments}

It is clear from figures 3 and 4 that the concentrated polymer solutions used in these experiments do not behave exactly like Newtonian fluids, but in fact are both shear thinning and viscoelastic. In the present section we therefore investigate the visco-elastocapillary thinning of adhesive filaments using the Giesekus model (Yao et al. 1998) and seek to understand the role of adhesive viscoelasticity on the thinning of adhesive filaments and their processability or tendency to form "strands".

We estimate the polymer contribution to the viscosity $\eta_{p}$ as the difference between the total adhesive viscosity $\eta(t)$, given by equation (13), and the constant solvent contribution to viscosity $\eta_{s}$. Hence, provided $\eta_{s} / \eta_{p}(t)<<1$ at all times, we obtain

$$
\frac{\eta_{p}(t)}{\eta_{0}\left(t_{0}\right)}=\exp \left(2 P \int_{0}^{t} S^{1 / 2} d t\right)-\beta_{s}
$$

where $\beta_{s}=\eta_{s} / \eta_{0}$ is the dimensionless solvent contribution to the initial viscosity. The change in the polymeric contribution to the viscosity arising from solvent evaporation also affects the relaxation behavior of the polymer chains. Again we have not directly measured the change in viscoelasticity with decreasing solvent concentration; however, in principle, this can be done. For the purpose of the simulation a simple analytic formulation consistent with the kinetic theory of polymer solutions can be obtained by using the following relationship

$$
\eta_{p}(z, t)=n(z, t) k T \lambda(z, t)
$$

where $k T$ is the thermal energy and $\lambda$ is the relaxation time. The number density of chains per unit volume, $n$, can be expressed in terms of the mass fraction of solvent as

$$
n(z, t)=\frac{(1-x(z, t)) \rho N_{A}}{M_{w}}
$$

where $\rho, N_{A}$ and $M_{w}$ are the density, Avagadro's number and the molecular weight of the polymer, respectively. Using equations (14), (15) and (16) we may evaluate the dimensionless relaxation time (or Elasto-capillary number $\Gamma=\lambda(z, t) \sigma / \eta_{0} R_{0}$ ) as

$$
\Gamma(z, t)=\Gamma_{0} \frac{1-x_{0}}{1-x_{0} \exp (-2 P y)} \frac{\exp (2 P y)-\beta_{s}}{1-\beta_{s}}
$$


Here, $y=\int_{0}^{t} S^{1 / 2} d t$ is a measure of the total stretch at time t. and, $\Gamma_{0}$ and $x_{0}$ are the initial elasto-capillary number and solvent mass fraction, respectively. Note that the elastocapillary number $\Gamma$ is a measure of the relative importance of the capillary necking rate to the stress relaxation rate in the filament. We can see from equation (17) that the elasticity of the polymer plays an increasingly important role on the thinning of the adhesive filament as the solvent evaporates. Also, in the limit $\Gamma \rightarrow 0$ the equations simplify to the Newtonian case discussed in the previous section. Table 3 presents approximate values of the initial elasto-capillary numbers $\Gamma_{0}$ for the various adhesive formulations studied in the present work.

Following Yao et al. (1998) the governing equations given by equations (2-6), for an adhesive filament described by the Giesekus model and undergoing elasto-capillary thinning can be written as

$$
\begin{aligned}
& 3 \beta_{s} \frac{\partial S}{\partial t}=f(t) S^{2}-\left(T_{z z}-T_{r r}\right) S-S^{3 / 2} \\
& \Gamma(z, t) \frac{\partial T_{z z}}{\partial t}=T_{z z}\left[2 \Gamma(z, t) \dot{\varepsilon}-1-\frac{\alpha \Gamma(z, t) T_{z z}}{\eta_{r}(z, t)}\right]+2 \eta_{r} \dot{\varepsilon} \\
& \Gamma(z, t) \frac{\partial T_{r r}}{\partial t}=-T_{r r}\left[\Gamma(z, t) \dot{\varepsilon}+1+\frac{\alpha \Gamma(z, t) T_{r r}}{\eta_{r}(z, t)}\right]-\eta_{r} \dot{\varepsilon}
\end{aligned}
$$

Here we have used the same dimensional scaling defined in the previous section, $T_{z z}$ and $T_{r r}$ are the $1 \mathrm{D}$ approximations of the polymeric stress, $\dot{\varepsilon}=1 / S(\partial S / \partial t)$ is the deformation rate of each slice, $\eta_{r}=\eta_{p}(z, t) / \eta_{0}$ and $\Gamma(z, t)$ is given by equation (17).

Since the total length of the filament is not changing with time, the tensile force in the filament can again be found from an integral constraint along the column as

$$
f(t)=\frac{\int_{0}^{\Lambda_{0}}\left[S\left(T_{z z}-T_{r r}\right)+S^{3 / 2}\right] d Z_{0}}{\int_{0}^{\Lambda_{0}} S^{2} d Z_{0}}
$$

Given an initial filament profile $R_{1}\left(Z_{0}, t_{1}\right)$ and an initial distribution of the polymeric stresses $T_{z z}\left(Z_{0}, t_{1}\right)$ and $T_{r r}\left(Z_{0}, t_{1}\right)$, eqs. (18-21) can be integrated in time to compute the 
evolution of the filament profile of a viscoelastic polymer solution undergoing viscoelasto-capillary thinning in the microfilament rheometer.

Figure 11 shows filament profiles for samples A5 and A2 at selective values of time. Initially, the filament profiles show remarkable similarity with the experimentally recorded images shown in figure 8 . Close to break-up the theoretical predictions do not exactly predict the experimentally observed axially uniform cylindrical threads held between large quasi-static or stagnant drops (or "blobs"). These differences in the evolution of the free surface profiles between the theory and experiments can be attributed to the one-dimensional model's inability to satisfy the no-slip boundary conditions at the end plates and the neglect of higher order corrections to the axial curvature. Hence, as the filament thins, some fluid slips radially outwards from the plate rather than making a stagnant drop or "blob" with the characteristic convex free surface profiles seen in figure 8 . For viscoelastic filaments with non-volatile solvents and no mass transfer, Yao et al. (1998) compared the predictions of this one-dimensional time dependent finite element simulations. They found that in the necked region away from the end plates the one-dimensional theory provides a very accurate description of the viscoelastic-capillary necking process and the time to break up. Since the principal quantitative measurement obtained in a microfilament rheometer is the midpoint radius $R_{\text {mid }}(t)$ we should expect the equation set (17)-(21) to provide a good description of the capillary thinning process in our adhesives without the need for a full numerical simulation.

The numerically computed evolution of the mid-filament diameter versus time are shown in figure 9 along with the experimentally measured data. Although the predictions are not completely quantitative for some samples, the predicted thinning of the adhesives samples shows almost the same trend as observed experimentally without any adjustment in any of the material parameters listed in Tables 1-3. The most viscoelastic and volatile samples A5 and A7 thin much more slowly compared to other samples, while the less viscoelastic samples such as A3, A2 and A6 exhibiting "good" processability neck down much faster than those samples exhibiting "O.K." processability (A4 and A1). Our inability to match exactly the theoretical predictions with the experimental observation profile is due to the number of simplifying assumptions made in developing the 1-D 
theory, however it is clear that we have captured the essential physical phenomena probed in the microfilament experiments. The principal experimental unknown is the precise value of the fluid interfacial tension which directly enters the capillary time scale $t_{c a p}$ used in non-dimensionalizing equations (18)-(21). The surface tension is assumed to be constant and equal to the solvent value. In reality, $\sigma$ will be a weakly time-varying function of the pure resin and solvent surface tension values, plus the mass fraction $x(t)$ of the solvent. Such an expression for the compositional variation in surface tension of the fluid can easily be incorporated in our model if values of the surface tension coefficients for mixtures of pure resin and solvent are known. Similarly, it is straightforward to perform multi-mode simulations, if desired, by duplicating the equations (19) - (20) for each additional viscoelastic mode with relaxation time $\lambda_{i}(z, t)$ and viscosity $\eta_{i}(z, t)$. The total polymeric stress obtained by summing the modes then appears in eq.(18).

\section{Discussion}

We have shown in the present work that the tendency of a pressure sensitive adhesive solution to form strands or exhibit "stringiness" cannot be readily deduced by conventional rheological tests that measure the viscometric properties of the polymer solution. Consequently, the processing characteristics of the material can not be readily correlated with familiar dimensionless groups such as the capillary number or Weissenberg number. However, direct measurement of the evolution of a slender filament in a microfilament rheometer indicates that the filament lifetime is a good measure of the processing characteristics of the viscoelastic material.

The dynamics of the filament evolution can be accurately modeled by a slender filament theory that incorporates the capillary driving force for breakup, the viscous (or viscoelastic) resistance from the extensional flow in the filament and the mass transfer of volatile components from the surface of the fluid. The material parameters characterizing each of these processes can all be directly measured using interfacial tensiometry $(\sigma)$, rheometry $(\eta(\dot{\gamma}))$ and thermogravimetric analysis $\left(h_{m}\right)$ respectively. The relative magnitude of these parameters for different fluid formulations can be expressed conveniently in terms of a single dimensionless group that quantifies the rate of mass 
transfer to the rate of capillary thinning and which we refer to as a Processability parameter $P=h_{m} \eta_{0} / \sigma$. For the polymeric fluids considered in the present study we find that materials with $P<3 \times 10^{-4}$ exhibit good processability, whilst material formulations with $P>10^{-3}$ show a strong tendency to form strands or exhibit "stringiness".

The small numerical values of the parameter $P$ reported here arise in part because we use the initial scales $\eta_{0}, R_{0}$ (and also $\lambda_{0}, \sigma_{0}$ ) in our analysis, whilst the actual physically-relevant scales change rapidly during the break-up process. In fact, the limit $P=0$ is a singular limit and a non-zero final filament radius is predicted in our present model for all $P \neq 0$, since the fluid viscosity $\eta(t)$ diverges as $x(t) \rightarrow 0$. However, the asymtotic value of the radius is given approximately by $R_{\infty} \cong R_{1} \exp (-0.0709 / 2 P)$ as we show in appendix A (see also figure 10) and becomes very small as $P \rightarrow 0$. For example, with an initial filament of $R_{1} \sim 1 \mathrm{~mm}$, the final thread radius becomes less than $1 \mu \mathrm{m}$ for $P \leq 5.1 \times 10^{-3}$. On such a scale, the presence of microscopic air bubbles, dust particles and other inclusions may cause the necking fluid column to rupture in a manner not captured by simple analysis of a homogeneous liquid thread. Furthermore the minimum object size that is optically detectable with our present laser micrometer is $2 R_{\min } \sim 4 \mu \mathrm{m}$ and thus we cannot observe the very final stages of the necking process.

The numerical simulations and experimental observations presented in this paper both show that as the parameter $P$ increases, the filament lifetime $t_{\text {life }}$ increases monotonically beyond the simple direct estimate of viscocapillary thinning, $t_{c a p} \sim \eta_{0} R_{0} / \sigma$. In a real commercial coating process, this filament lifetime must be compared with a characteristic shear rate $\dot{\gamma}$ or a residence time, $t_{\text {res }} \sim 1 / \dot{\gamma}$. If the residence time of a fluid element in the flow is of the same order as, or greater than, the filament lifetime $t_{\text {life }}(P)$ then the formation and growth of strands or "stringiness" is likely. Physically, viscous and/or elastic forces retard the capillary necking and breakup of the filament long enough for significant solvent evaporation to occur, this in turn increases the viscous and elastic material properties which feed back to further retard the necking and breakup of the thread. This simple argument (based on visco-capillary thinning of a Newtonian filament) suggests that stranding can be avoided by simply increasing the characteristic shear rate of the process. However, for a real pressure 
sensitive adhesive formulation consisting of a concentrated polymer solution in a volatile solvent the filament lifetime is also a function of the initial elastic stress difference $\left(T_{z z}-T_{r r}\right)_{0}$ in the fluid and the elastocapillary number $\Gamma=\lambda(z, t) \sigma / \eta_{0} R_{0}$. The simple onedimensional model presented here can be used to perform numerical simulations of $t_{\text {life }}\left(P, \Gamma_{0},\left(T_{z z}-T_{r r}\right)_{0}\right)$ and the resulting values can be compared with the residence time $t_{r e s}$ to understand the processability characteristics of a viscoelastic fluid.

Finally, we note that the theoretical analysis and experimental method presented in this paper can also be adapted readily to consider the case of combined heat transfer and visco-elasto-capillary necking - a process commonly encountered during batch filling operations in the food and consumer products industries. In this case, the rate of mass transfer $\left(h_{m} / R_{0}\right)$ is replaced by the corresponding rate of heat transfer $\left(h / \rho c_{p} R_{0}\right)$ which can be obtained from an energy balance combined with Newton's law of cooling (i.e. the analogous equation to equation (1)). The non-isothermal analog of the processability parameter $P$ is then $h \eta_{0} / \sigma \rho c_{p}$ (where $h$ is the heat transfer coefficient and $c_{p}$ is the specific heat). By analogy with equation (11), this dimensionless grouping can also be expressed in terms of the Nusselt, Prandtl, Reynolds and capillary numbers. The corresponding non-isothermal viscoelastic constitutive equation required for visco-elastocapillary breakup with combined heat transfer can be obtained using the "psuedotime hypothesis' (see for example Tanner, 1985). For a thermorheologically simple material, the variation in the material properties $\eta(t)$ and $\lambda(t)$, as the filament necks and cools, can simply be expressed in terms of the standard rheological shift factor $a\left(T(t), T_{0}\right)$ determined using time-temperature superposition. For many materials the thermal variation in surface tension $d \sigma / d T$ is also known and can also be simply incorporated in the model. We hope to explore the effect of heat transfer on capillary breakup and the lifetime of necking filaments further in the future.

\section{Acknowledgments}

AT and GHM would like to thank Dupont corporation and the Lord Foundation for financial support of this research. 


\section{Appendix A}

In this appendix we show that a reasonable analytical approximation to the equation set (7), (8) and (13) can be obtained by ignoring all axial free surface variations. In this limit the slender fluid element becomes a viscous thread with $R(z, t) \cong R(t)$ and setting the tensile force $F$ in equation (5) to $2 X \pi R \sigma$, we obtain (see Mckinley \& Tripathi, 1999)

$$
3 \eta(t)\left(-\frac{2}{R(t)} \frac{d R(t)}{d t}\right)=\frac{\sigma}{R(t)}(2 X-1)
$$

where $X$ is the correction factor to the tensile force arising from weak axial variation in $R(t)$ and its value is close to 0.7127 (Papageorgiou, 1995; McKinley \& Tripathi, 1999). Note that $X=1$ and $\eta(t)=\eta_{0}$ corresponds to the equation derived by Entov \& Hinch (1997). On substituting for the time-dependent viscosity function from equation (13) and then non-dimensionalizing, equation (A1) can be expressed as a nonlinear second order ODE given by

$$
y^{\prime \prime}=0.0709\left(y^{\prime}\right)^{2} \exp \{-2 P y\}
$$

where $y^{\prime}=R_{0} / R(t)$. Equation (A2) can be readily integrated (Dr. Aaron Avagliano, Personal Communication) using initial conditions at $y^{\prime}(t=0)=R_{0} / R_{1}, y(t=0)=0$ to obtain the implicit solution

$$
t=\frac{\eta_{0} R_{1}}{\sigma} \int_{0}^{y} \exp \left[\frac{0.0709}{2 P}(\exp (-2 P y)-1)\right] d y \text { for } \quad P \neq 0
$$

Note that for all $P \neq 0$ this simple analytical model predicts a finite final thread radius $R_{\infty} \cong R_{1} \exp (-0.0709 / 2 P)$ since the viscosity diverges as the thread radius and solvent mass fraction approach zero. This final thread radius is indicated by the asymptotic values of the dotted lines in figure 10. In the limit $P=0$, expansion of the integrand in equation (A3) or direct integration of equation (A1) gives

$$
\frac{R(t)}{R_{0}}=\frac{R_{1}}{R_{0}}-0.0709 \frac{\sigma}{R_{0} \eta_{0}} t
$$

so that the critical time to break up is $t_{c}=14.104 \eta_{0} R_{1} / \sigma$, in agreement with experimental observations. 


\section{References}

1. A. Acrivos, M. J. Shah, and E. E. Petersen, "On the flow of a non-Newtonian liquid on a rotating disk," J. Appl. Phys. 31, 963 (1960).

2. A. V. Bazilevsky, V. M. Entov, and A. N. Rozhkov, "Liquid filament microrheometer and some of its applications," In D. R. Oliver (Ed.), Proc. Third European Rheology Conference (Elsevier, London and New York), 41 (1990).

3. L. Benyahia, C. Verdier and J. M. Piau, "The mechanism of peeling of uncross-linked pressure sensitive adhesives," Journal Adhesion 62, 45 (1997).

4. R. B. Bird, R. C. Armstrong and O. Hassager, Dynamics of Polymeric liquids, Vol 1, Fluid Mechanics, Wiley Interscience, New York, (1987).

5. R. B. Bird, W.E. Stewart and E. N. Lightfoot, Transport Phenomena, John Wiley, New York, (1960).

6. D. P. Birnie and M. Manley, "Combined flow and evaporation of fluid on a spinning disk," Phys. Fluids 9(4), 870 (1997).

7. D.W. Bousfield, R. Keunings, G. Marrucci and M. M. Denn, "Nonlinear Analysis of the Surface-Tension Driven Breakup of Viscoelastic Fluid Filaments," J. Non-Newt. Fluid Mech., 21, 79 (1986).

8. J. A. Britten and I. A. Thomas, "Non-Newtonian flow effects during spin coating large-area optical coatings with colloidal suspensions," J. Appl. Phys. 71, 972 (1992).

9. E. P. Chang, "Viscoelastic windows of presssure-sensitive adhesives," J. Adhesion 34 (1-4), 189 (1991).

10. S. F. Christensen and G. H. McKinley, "Rheological modeling of the peeling of pressure-sensitive adhesive and other elastomers," Int. J. Adhesion \& Adhesive 18(5), 333 (1998).

11. R. W. Connelly, W. F. Parsons and G. H. Pearson, "Prediction of peel adhesion using extensional rheometry," J. Rheol. 25, 315 (1981); see also R. K. Gupta, "Comments on "prediction of peel adhesion using extensional rheometry" by Connelly, Parsons and Pearson,” J. Rheol. 27(2), 171 (1983).

12. D. J. Coyle, C. W. Macosko and L. E. Scriven, "Reverse roll coating of nonNewtonian liquids,” J. Rheol. 34(5), 615 (1990). 
13. C. Derail, A. Allal, G. Martin and P. Tordjeman, "Relationship between viscoelastic and peeling properties of model adhesives. Part I. Cohesive fracture," J. Adhesion 61(1-4), 123 (1997).

14. J. Eggers, "Nonlinear dynamics and breakup of free-surface flows," Rev. Mod. Phys. 69(3), 865 (1997).

15. V. M. Entov and E. J. Hinch, "Effect of a spectrum of relaxation time on the capillary thinning of a filament of elastic liquid," J. Non-Newtonian Fluid Mech. 72, 31 (1997).

16. M. H. Eres, D. E. Weidner, and L. W. Schwarz, "Three-dimensional direct numerical simulation surface tension gradient effects on the leveling of an evaporating multicomponent fluid," Langmuir 15, 1859 (1999).

17. J. Ferguson, B. Reilly, and N. Granville, "Extensional and adhesion characteristics of a pressure sensitive adhesive," Polymer 38, 795 (1997).

18. W. R. Gambit, "How to estimate mixture viscosities," Chem. Engg. 66(3), 151 (1959).

19. S. Gaudet, G. H. McKinley, and H. A. Stone, "Extensional deformation of Newtonian liquid bridges," Phys. Fluids 8(10), 2568 (1996).

20. R. D. Gillete and D. C. Dyson, "Stability of fluid interfaces of revolution between equal solid circular plates," Chem. Eng. J. 2, 44 (1971).

21. R. K. Gupta, "Extensional rheometry of polymer melts," in "Adhesive bonding" ed. L. H. Lee, Plenum press, NY (1991).

22. R. K. Gupta and T. Sridhar, in A. A. Collyer and D. W. Clegg (Eds.), "Rheological measurement," Elsevier Applied Science, London (1988).

23. O. Hassager, M. I. Kolte, M. Renardy, "Failure and nonfailure of fluid filaments in extension,” J. Non-Newtonian Fluid Mech. 76, 137 (1998).

24. S. D. Howison, J. A. Moriarty, J. R. Ockendon, E. L. Terrill, and S. K. Wilson, “A mathematical model for drying paint layers,” J. Engg. Math. 32, 377 (1997).

25. J. B. Irving, "Viscosity of binary liquid mixtures: A survey of mixture equations," NEL Report, 630, Feb (1977).

26. D. F. James and K. A. Walters, “Techniques in rheological measurements," Elsevier, London (1993). 
27. S. Kojima, T. Moriga, and K. Takenouchi, "The leveling of thermosetting waterborne coatings. Part 3: Leveling under controlled conditions," Polym. Eng. Sci. 35, 1949 (1995).

28. M. I. Kolte and P. Szabo, "Capillary thinning of polymeric filaments," J. Rheol. 43(3), 609 (1999).

29. H. Lakrout, P. Sergot and C. Creton, "Direct observations of cavitation and fibrillation in a probe tack experiment on model acrylic pressure sensitive adhesive," J. of Adhesion 69, 307 (1999).

30. R. G. Larson, "Spinnability and viscoelasticity,” J. Non-Newtonian Fluid Mech.12, 303 (1983).

31. C. J. Lawrence and W. Zhou, "Spin coating of non-Newtonian fluids," J. NonNewtonian Fluid Mech. 39, 963 (1991).

32. J. M. Li, W. R. Burghardt, B. Yang and B. Khomami, "Flow birefringence and computational studies of a shear thinning polymer solution in axisymmetric stagnation flow,” J. Non-Newtonian Fluid Mech. 74, 151 (1998).

33. R. F. Liang and M. R. Mackley, "Rheological characterization of the time and strain dependence for polyisobutylene solutions," J. Non-Newtonian Fluid Mech. 52, 387 (1994).

34. G. H. McKinley and A. Tripathi, "Extracting the Newtonian Viscosity from observations of Capillary Breakup in a Filament Rheometer," J. Rheol, submitted, 1999

35. R. Ober, L. Paz, C. Taupin and P. Pincus, "Study of the surface tension of polymer solutions - theory and experiments. 1. Good solvent conditions," Macromolecules 16(1), 50 (1983).

36. W. S. Overdiep, “The leveling of paints,” Prog. in Org. Coatings 14, 159 (1986).

37. R. Padmanabhan, "Measurement of extensional viscosity of viscoelastic liquid foods," J. Food Engineering 25(3), 311 (1995).

38. D. T. Papageorgiou, "On the breakup of viscous liquid threads," Phys. Fluids 7(7), 1529 (1995).

39. J. R. A. Pearson, Mechanics of Polymer Processing, Elsevier, London, 1985. 
40. J. M Piau, C. Verdier and L. Benyahia, "Influence of rheology and surface properties in the adhesion of uncross-linked pressure sensitive adhesives," Rheol. Acta 36, 449 (1997).

41. M. Renardy, "A numerical study of the asymptotic evolution and breakup of Newtonian and viscoelastic jets," J. Non-Newtonian Fluid Mech. 59, 267 (1995).

42. L. A. Slobozhanin and J. M Perales, "Stability of liquid bridges between equal disks in an axial gravity field," Phys. Fluids A 5, 1305 (1993).

43. S. H. Spiegelberg, D. C. Ables and G. H. McKinley, "The role of end-effects on measurements of extensional viscosity in filament stretching rheometers," J. NonNewtonian Fluid Mech. 64, 229 (1996).

44. R. I. Tanner, Enginnering Rheology, Claredon Press, Oxford (1985).

45. S. Taneya, T. Izutsu, T. Kimura, T. Shioya, W. Buchheim, P. S. Kindstedt and D.G. Pechak, "Structure and rheology of string cheese," Food Structure 11, 61 (1992).

46. F. T. Trouton, "On the coefficient of viscous traction and its relation to that of Proc. R. Soc. London Ser A 77, 426 (1906).

47. Y. Urahama, "Effect of peel load on stringiness phenomenon and peel speed of pressure-sensitive adhesive tape," J. Adhesion 31, 47 (1989).

48. S. M. Wang, B. M. Watts, O. M. Lukow, L. Schlichting and W. Bushuk, "Dough profiling: An instrumental method for dough stickness measurement," Cereal Chemistry 73(4), 445 (1996).

49. M. Yao, G. H. McKinley, and B. Debbaut, "Extensional deformation, stress relaxation and necking failure of viscoelastic filaments," J. Non-Newtonian Fluid Mech. 79, 469 (1998).

50. A. Ziabicki, The Fundamentals of Fiber Formation: The Science of Fiber Spinning and Drawing, Wiley Interscience, New York, 1976.

51. A. Zosel, "The effect of fibrilation on the tack of pressure sensitive adhesives," Int. J. Adhesion and Adhesives 18(4), 265 (1998). 


\section{Captions}

Figure 1: Representative geometries for important commercial processes in which both the Non-Newtonian rheology of the bulk fluid and mass transfer of a volatile solvent from the free surface are important: (a) leveling, drainage and sagging of paint films; (b) spin coating of resists over non-uniform surface topologies; (c) viscous withdrawal of sheets and wires; (d) roll-coating of pressure sensitive adhesives.

Figure 2: Solvent evaporation as a function of time for seven adhesive formulations. The experimental data was obtained using a thermogravimetric analyzer (TGA). The solid lines show three (A1, A2 \& A6) of the exponential fits obtained using equation (1).

A1; - - - - A2; - - - - A6. Values of the fitted mass transfer coefficient are given in Table 1.

Figure 3: Steady shear viscosity as a function of shear rate, $\eta(\dot{\gamma})$, and non-linear fits using single-mode Giesekus model. The fits are shown for three (A1, A2 \& A6) of the adhesive samples. $\mathrm{A} 1 ;-----\mathrm{A} 2 ;-\cdot-\cdot-\cdot \mathrm{A} 6$.

Figure 4: First normal stress coefficient as a function of shear rate, $\Psi_{1}(\dot{\gamma})$, and non-linear fits using single-mode Giesekus model. The fits are shown for three (A1, A2 \& A6) of the adhesive samples. $\longrightarrow \mathrm{A} 1 ;-----\mathrm{A} 2 ;-\cdot-\cdot-\cdot \mathrm{A} 6$.

Figure 5: Capillary number $C a=\eta \dot{\gamma} R_{0} / \sigma$ as a function of shear rate for various adhesive samples. The solid lines correspond to the capillary number evaluated using the fitted viscosity function obtained from the Giesekus model. $\mathrm{A} 1 ;-----\mathrm{A} 2$; $-\cdot-\cdot-\cdot$ A6.

Figure 6: Variation of the Weissenberg number, $W i=N_{1} / 2 \tau_{12}$, as a function of shear rate for various adhesive samples. The solid lines represent the estimated Weissenberg number using model fitted values. —— A1; - - - - A2; - - - - . A6. Figure 7: Schematic diagram of Micro-Filament Rheometer (MFR): (a) fixed lower plate; (b) movable upper plate; (c) spring assembly; (d) solvent trap; (e) CCD camera; (f) laser micrometer; (g) laser light (h) syringe injection system (i) background illumination. 
Figure 8: Two samples (A2 \& A5) undergoing visco-elasto-capillary thinning at temperature $T \cong 25^{\circ} \mathrm{C}$. Consecutive images are recorded by a CCD camera in the microfilament rhometer. The two sets of images show a clear visual distinction between fluids exhibiting "BAD”(A5) and "GOOD”(A2) processing characteristics.

Figure 9: The transient midpoint filament diameter profiles for each fluid formulation measured in the micro-filament rheometer. The experimental data is shown using filled symbols and the predictions of the Giesekus model (equations (18-21)) are shown using lines and the corresponding hollow symbols. Numerical values of the dimensionless parameters characterizing the individual materials and employed in the simulations are given in table 3 . The experimental profiles clearly separate the materials exhibiting "GOOD", "BAD” and "O.K." processing characteristics into different regions of the graph.

Figure 10: The predictions of the transient mid-plane radius obtained numerically from the Newtonian model for Processability parameters $P=0,0.005,0.01,0.05$. The plot also includes the analytical solution obtained using a cylindrical thread theory described in appendix A.

Figure 11: Numerically predicted filament profiles for samples A5 and A2 at selective values of time. These profiles were computed using the Giesekus filament model with parameter values given in table 3 . 
Table 1: Solvent composition, mass transfer characteristics and "processability" of seven adhesive formulations studied in this paper

\begin{tabular}{|c|l|c|c|c|}
\hline $\begin{array}{c}\text { Adhesive } \\
\text { sample }\end{array}$ & Solvent & Processability $^{(i)}$ & $\begin{array}{c}\text { Initial solvent } \\
\text { mass fraction }\end{array}$ & $\begin{array}{c}\text { Mass transfer coefficient } \\
h_{m} A / V\left[s^{-1}\right] \times 10^{3}\end{array}$ \\
\hline \hline A1 & Heptane & OK & 0.34 & 0.467 \\
A2 & Heptane & Good & 0.63 & 0.621 \\
A3 & Heptane & Good & 0.65 & 0.824 \\
A4 & Heptane & OK & 0.37 & 0.984 \\
A5 & Hexane & Bad & 0.33 & 1.338 \\
A6 & Heptane & Good & 0.33 & 1.602 \\
A7 & Hexane & Bad & 0.32 & 1.879 \\
\hline
\end{tabular}

(i) As indicated by the supplier; determined by the tendency of fluid sample to form "strands" or appear stringy. 
Table 2: Rheological parameters of the seven adhesive samples obtained from steady shear rheometry.

\begin{tabular}{|c|c|l|l|c|c|}
\hline $\begin{array}{c}\text { Adhesive } \\
\text { sample }\end{array}$ & $\begin{array}{c}\text { Zero shear-rate } \\
\text { viscosity }{ }^{(\mathrm{i})} \\
\eta_{0}[\mathrm{~Pa} . \mathrm{s}]\end{array}$ & $\begin{array}{l}\text { Relaxation } \\
\text { time } \\
\lambda[\mathrm{s}]\end{array}$ & $\begin{array}{c}\text { Mobility } \\
\text { factor } \\
\alpha[-]\end{array}$ & $\begin{array}{c}\text { Solvent } \\
\text { viscosity } \\
\eta_{s}[\mathrm{~Pa} . \mathrm{s}]\end{array}$ & $\begin{array}{c}\text { Surface } \\
\text { tension } \\
\sigma[\mathrm{N} / \mathrm{m}]\end{array}$ \\
\hline \hline A1 & 17.0 & $1.88 \times 10^{-1}$ & 0.43 & 1.50 & $2.03 \times 10^{-2}$ \\
A2 & 2.0 & $5.70 \times 10^{-2}$ & 0.48 & 0.34 & $2.03 \times 10^{-2}$ \\
A3 & 2.0 & $3.02 \times 10^{-2}$ & 0.20 & 0.16 & $2.03 \times 10^{-2}$ \\
A4 & 14.0 & $2.07 \times 10^{-1}$ & 0.45 & 1.40 & $2.03 \times 10^{-2}$ \\
A5 & 19.0 & $5.73 \times 10^{-1}$ & 0.05 & 1.90 & $1.79 \times 10^{-2}$ \\
A6 & 0.8 & $2.85 \times 10^{-3}$ & 0.46 & 0.23 & $2.03 \times 10^{-2}$ \\
A7 & 15.0 & $4.09 \times 10^{-1}$ & 0.10 & 0.90 & $1.79 \times 10^{-2}$ \\
\hline
\end{tabular}

(i) The zero shear-rate viscosity of the Giesekus model is $\eta_{0}=\eta_{s}+\eta_{p}$. 
Table 3: The estimated values of the Processability parameter $P$ and the Elasto-capillary number for various adhesive formulations along with their "processability".

\begin{tabular}{|c|c|c|c|}
\hline $\begin{array}{c}\text { Adhesive } \\
\text { sample }\end{array}$ & Processability & $\begin{array}{c}\text { Parameter } \\
P=h_{m} \eta_{0} / \sigma\end{array}$ & $\begin{array}{c}\text { Elasto-capillary Number } \\
\Gamma_{0}=\lambda \sigma / \eta_{0} R_{0}\end{array}$ \\
\hline \hline A6 & Good & $1.78 \times 10^{-4}$ & 0.03 \\
A2 & Good & $1.83 \times 10^{-4}$ & 0.19 \\
A3 & Good & $2.43 \times 10^{-4}$ & 0.10 \\
A1 & OK & $1.17 \times 10^{-3}$ & 0.07 \\
A4 & OK & $2.04 \times 10^{-3}$ & 0.10 \\
A5 & Bad & $4.26 \times 10^{-3}$ & 0.18 \\
A7 & Bad & $4.72 \times 10^{-3}$ & 0.16 \\
\hline
\end{tabular}


a)

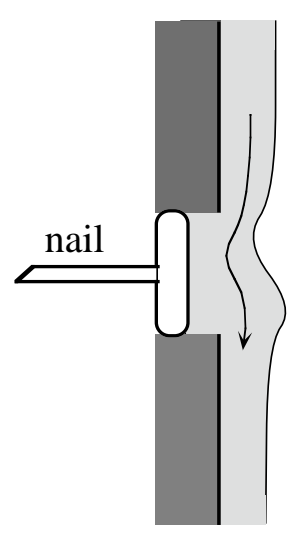

c)

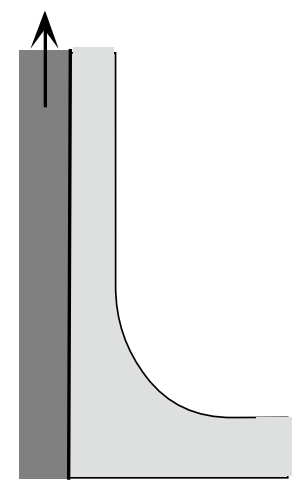

b)

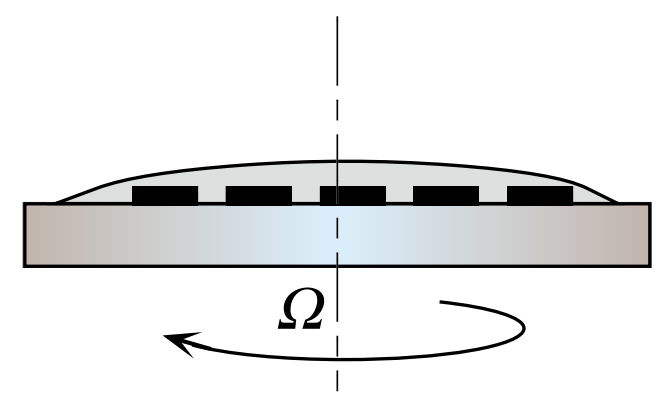

d)

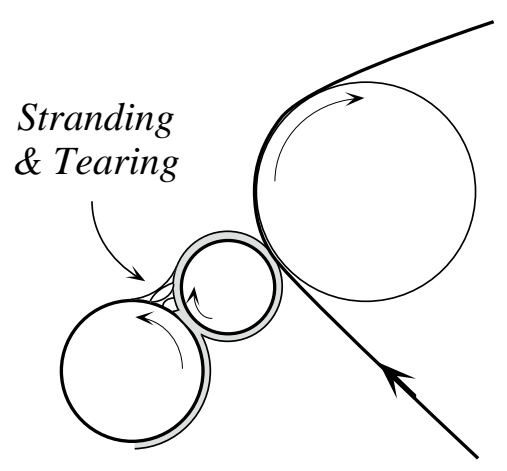

Figure 1 


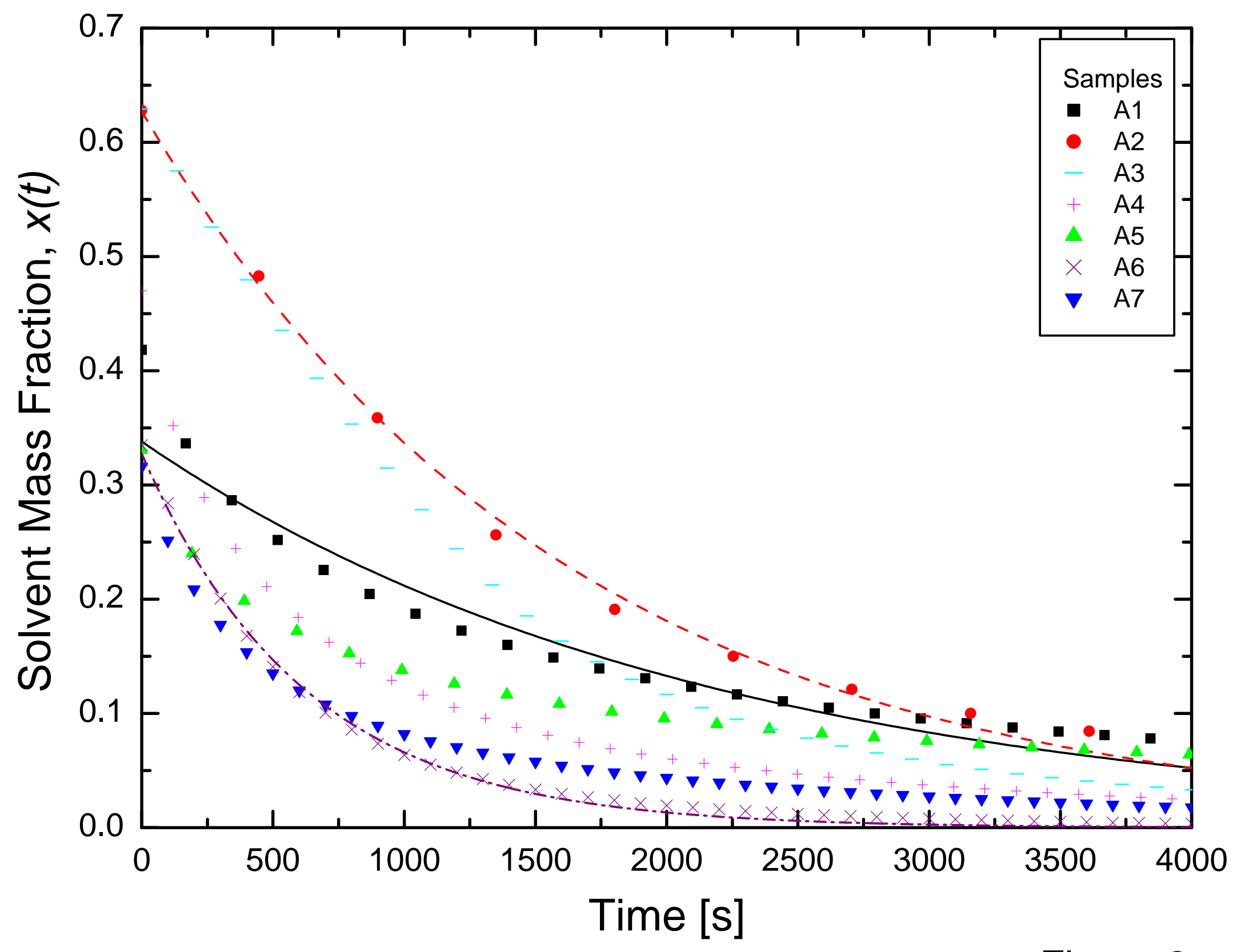

Figure 2 


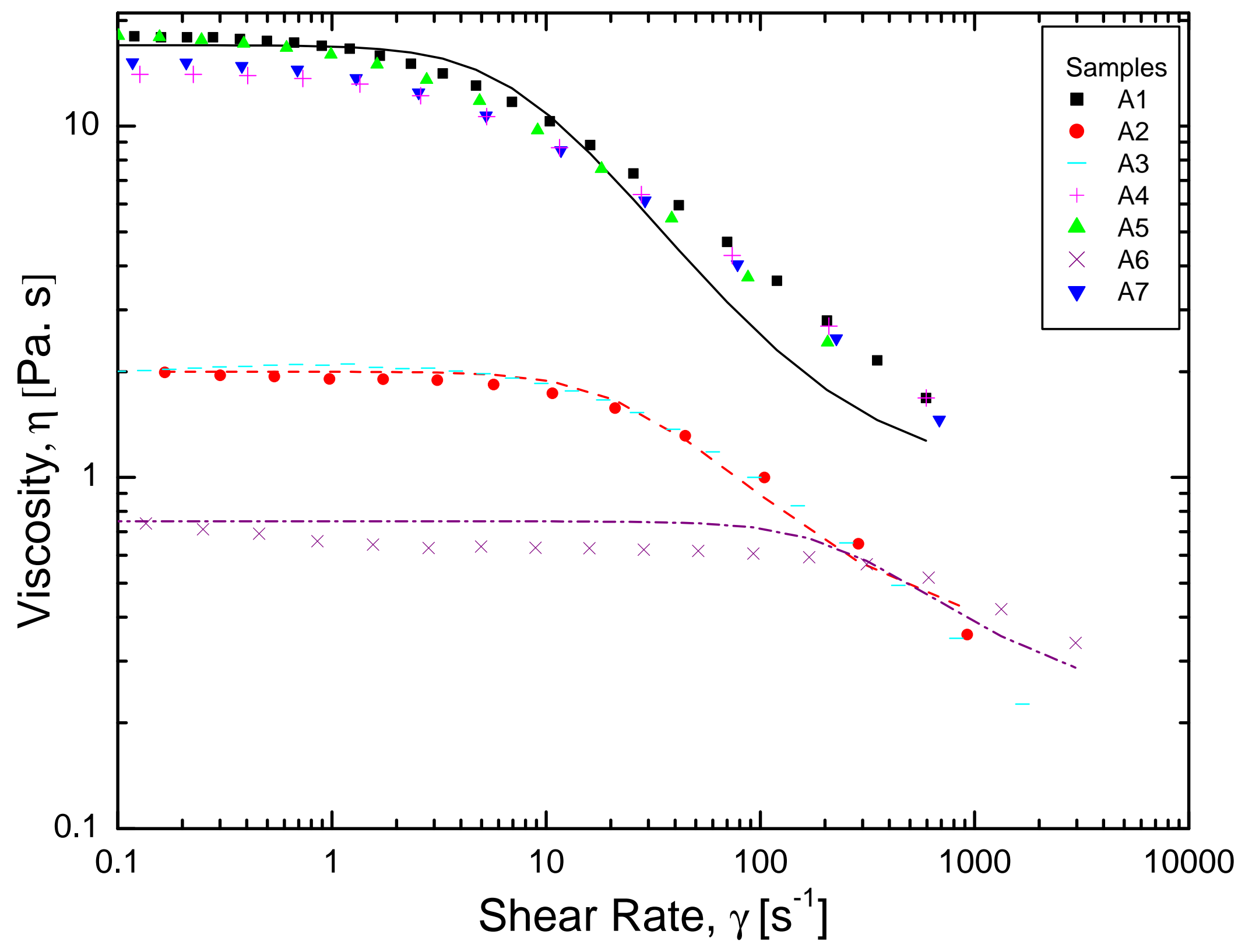

Figure 3 


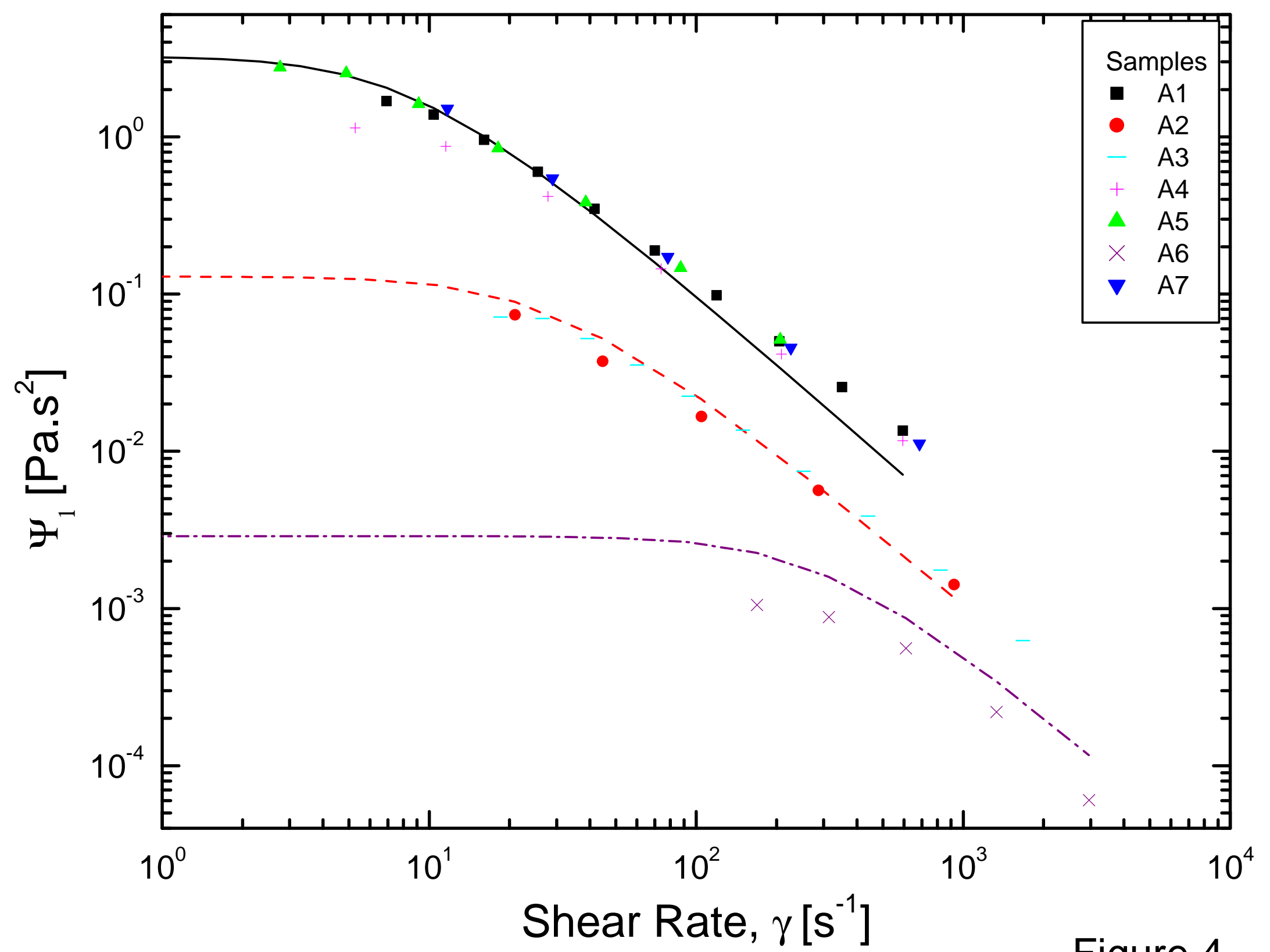

Figure 4 


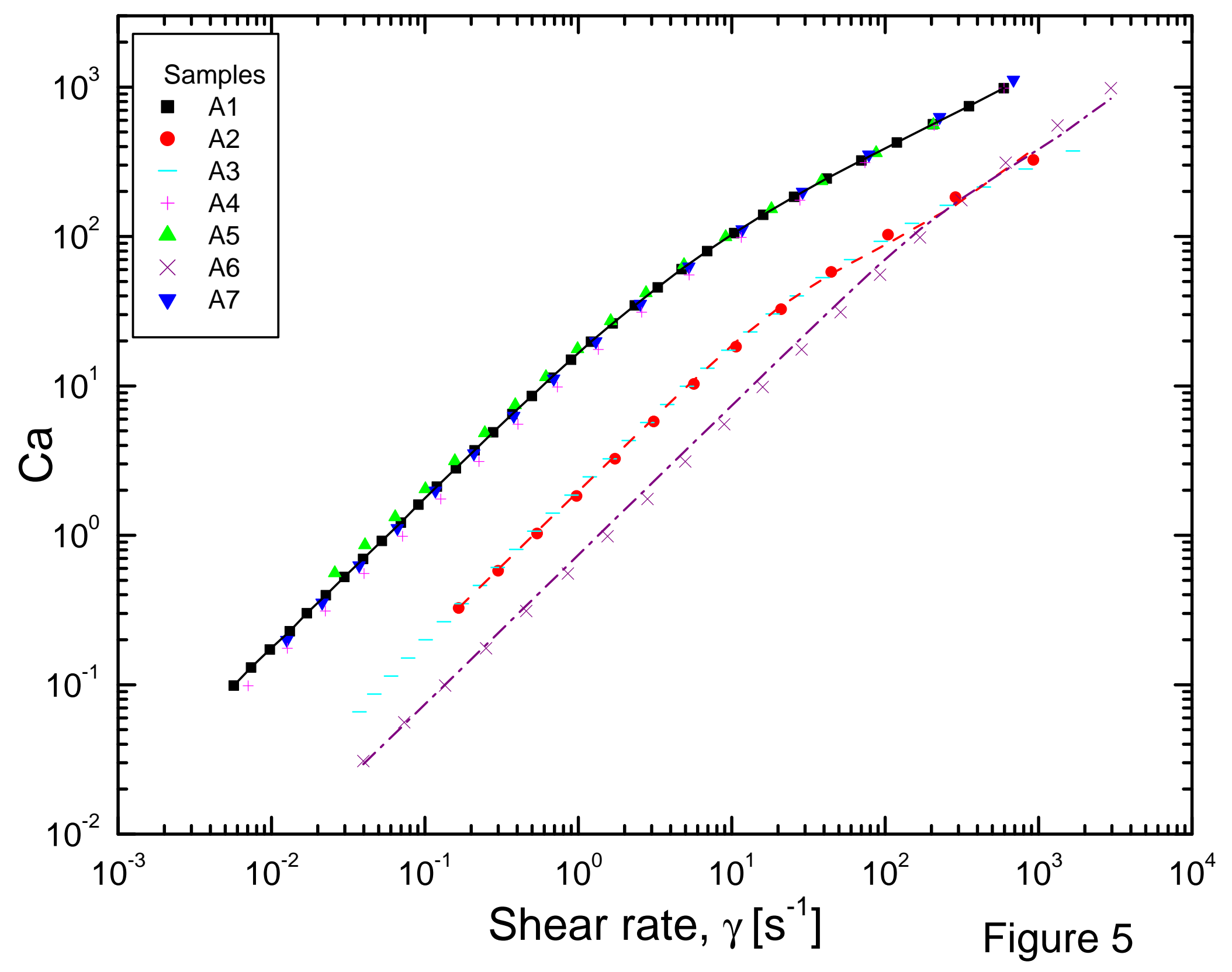




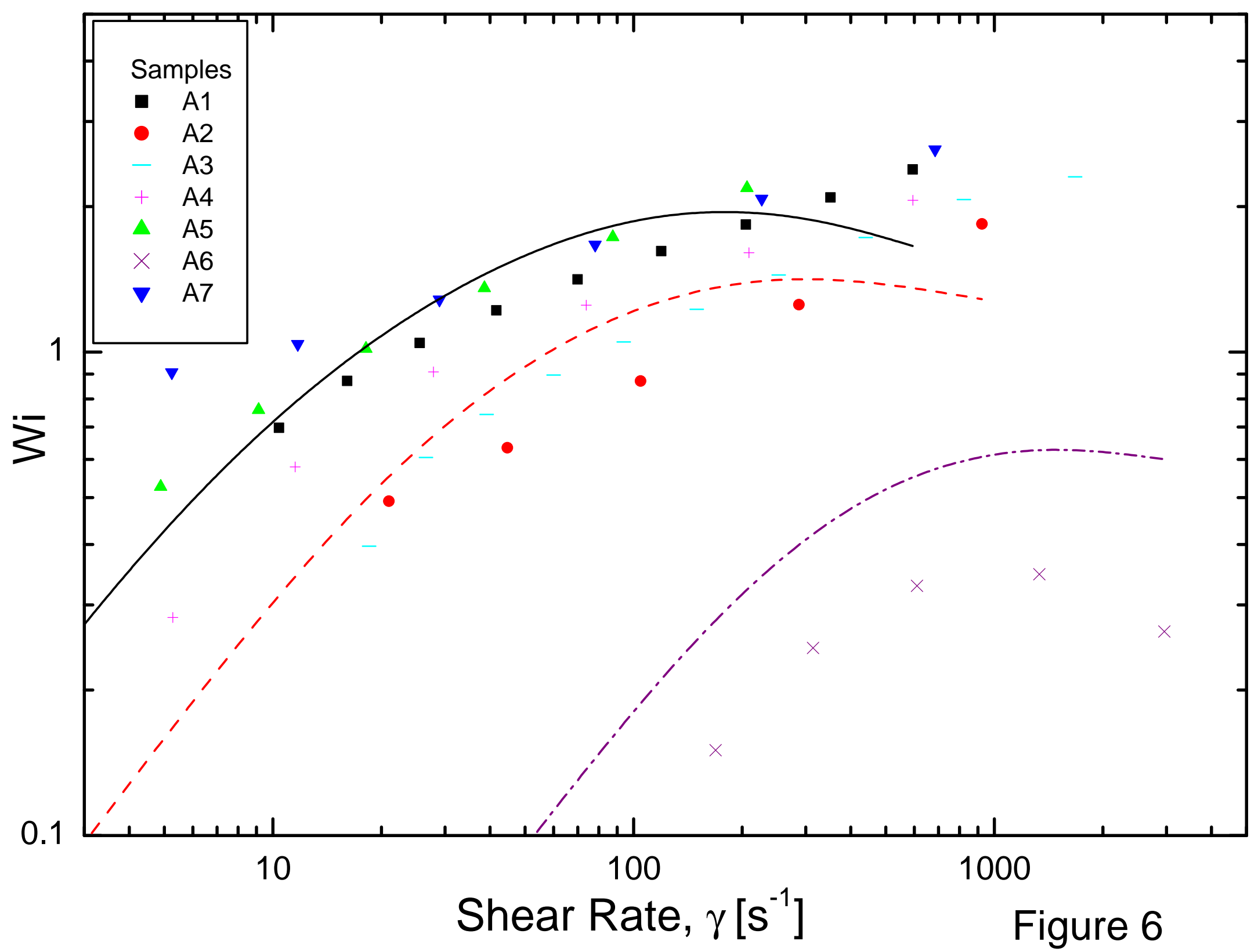




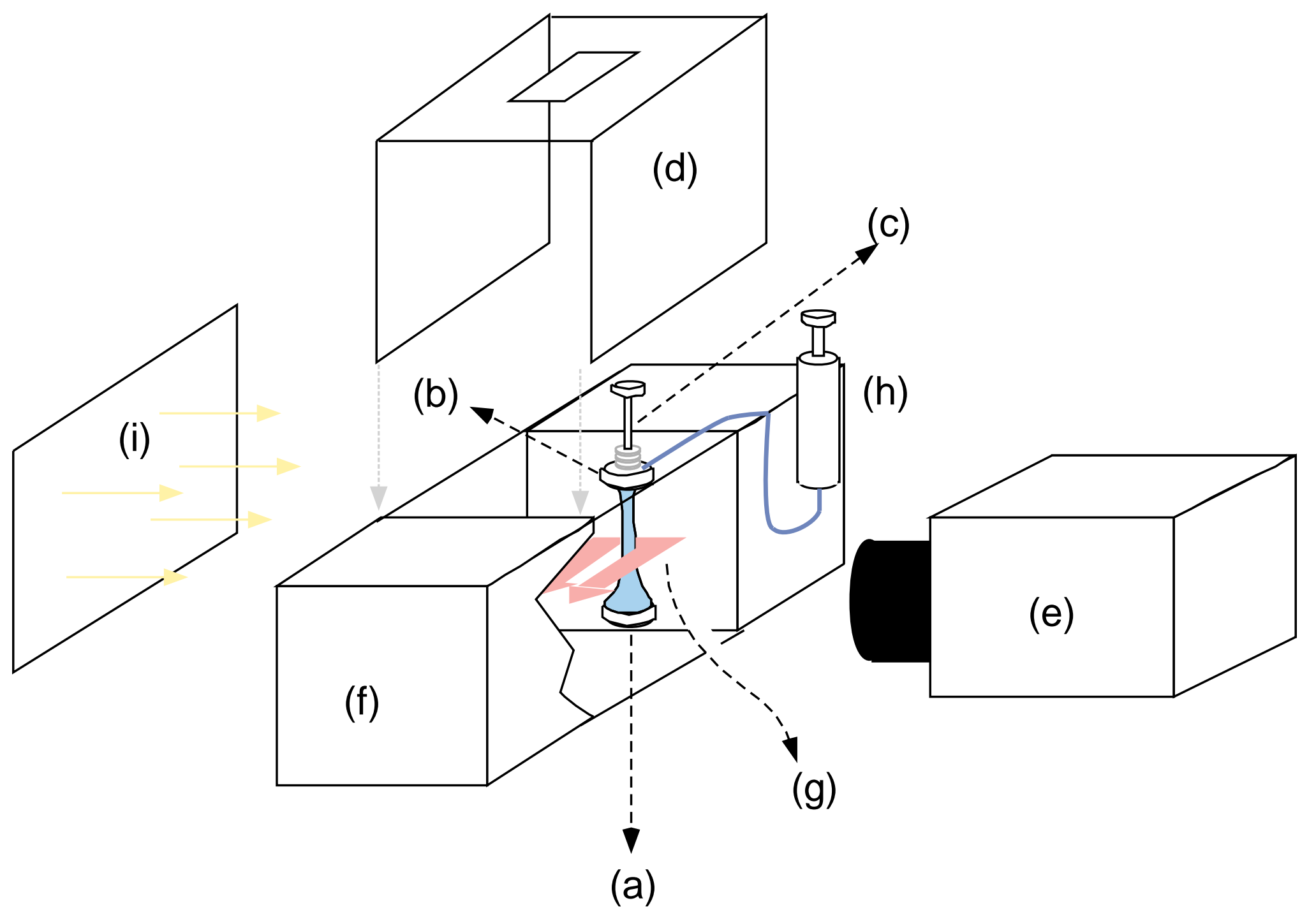


A2
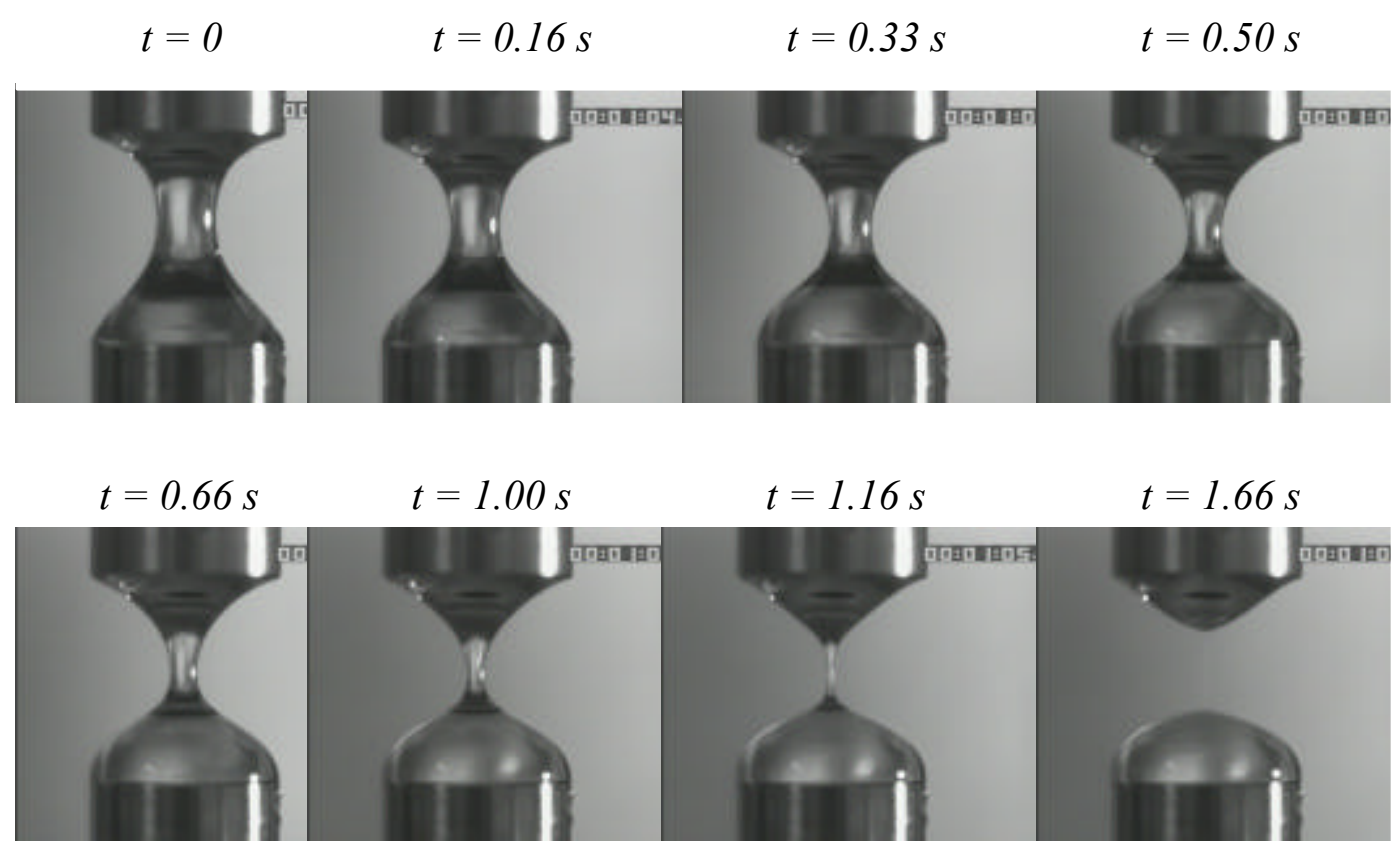

A5

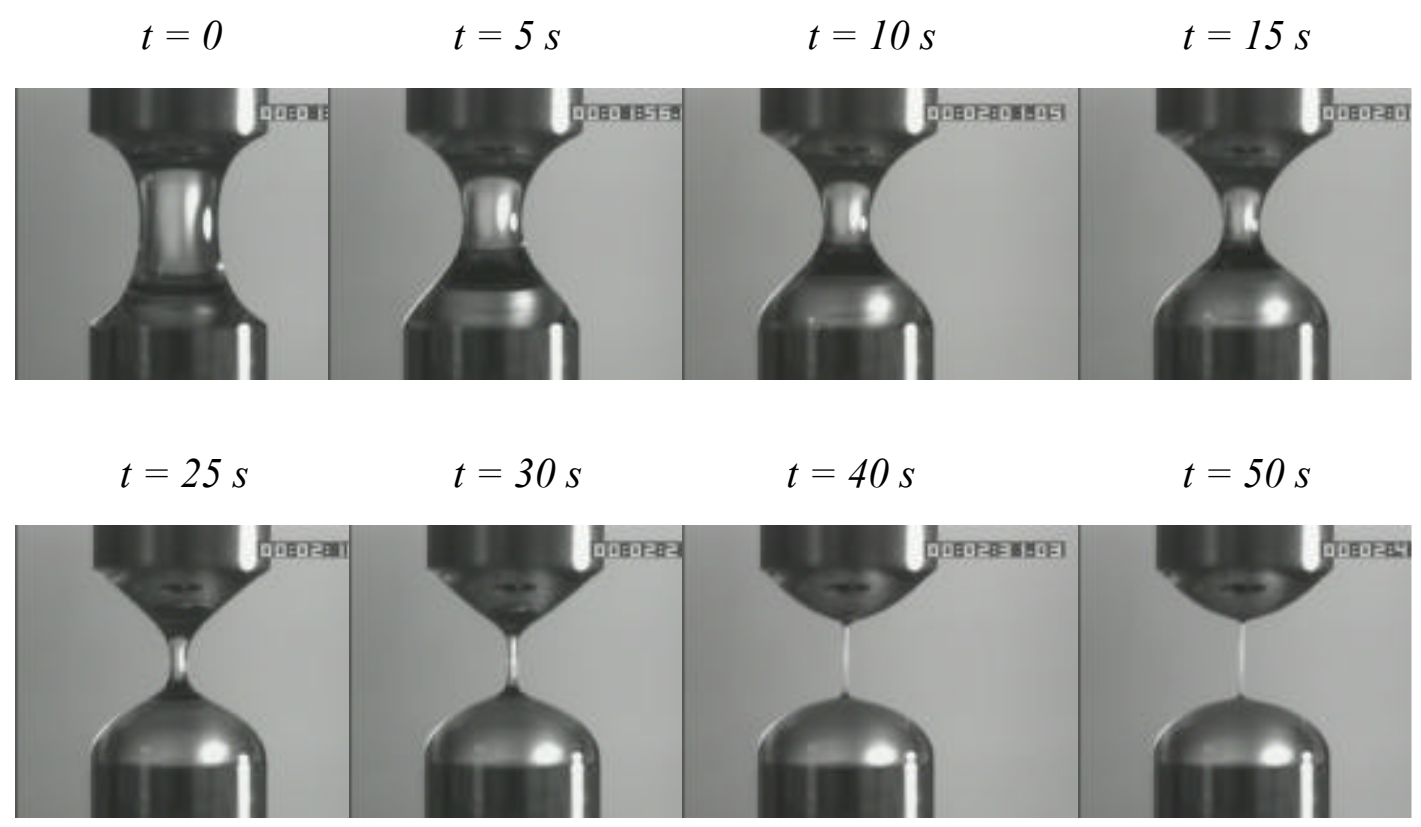

Figure 8 


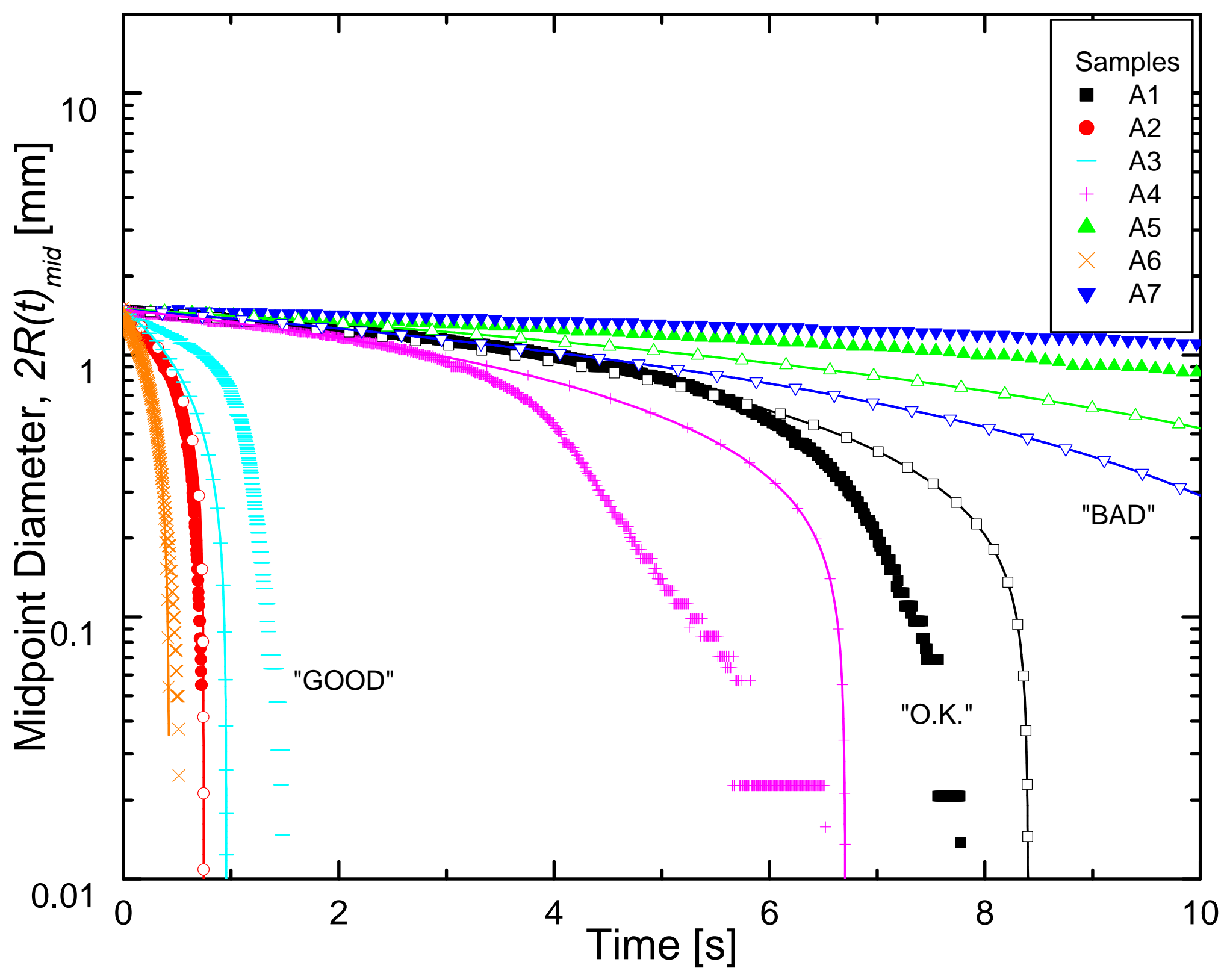

Figure 9 


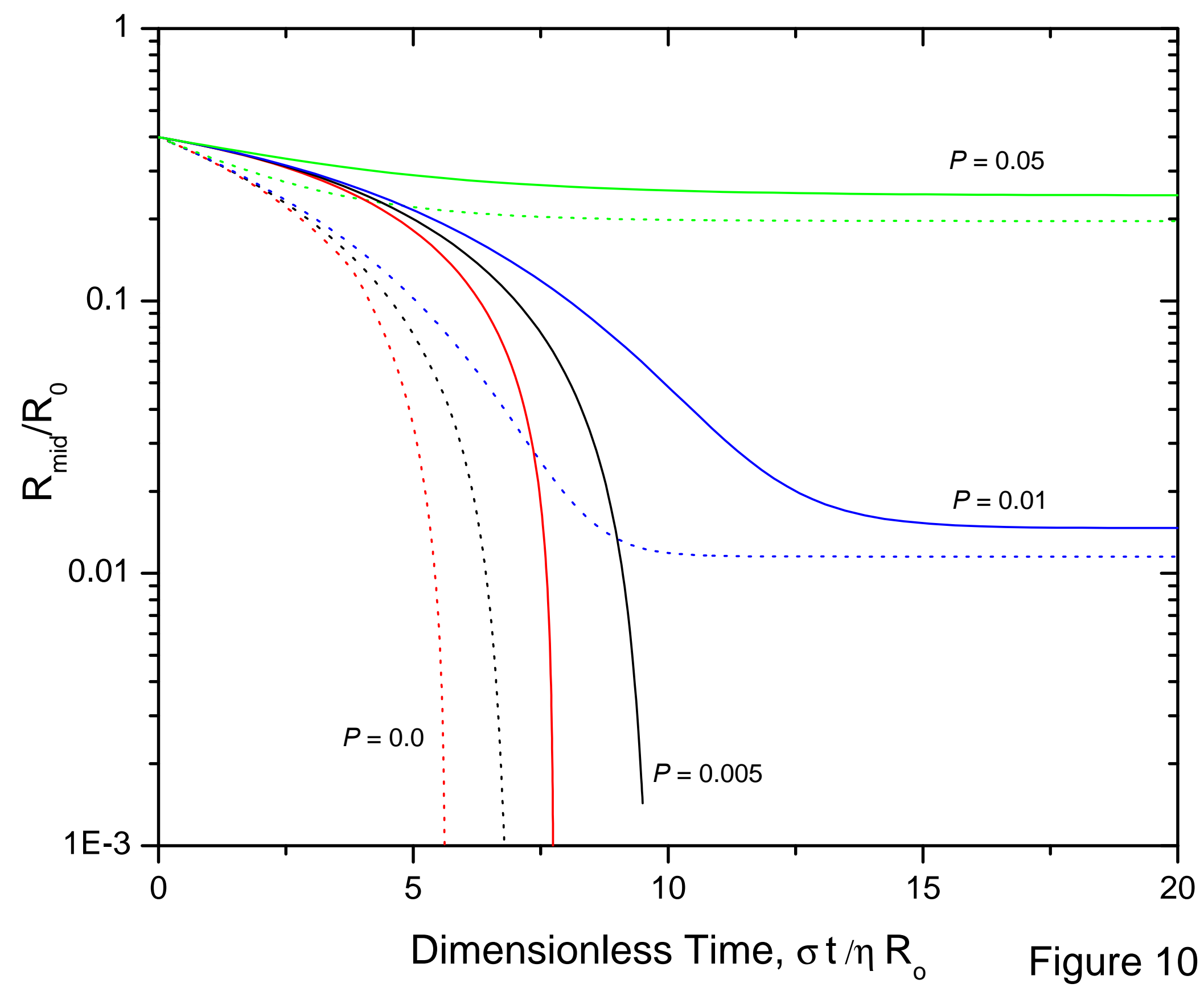


A2
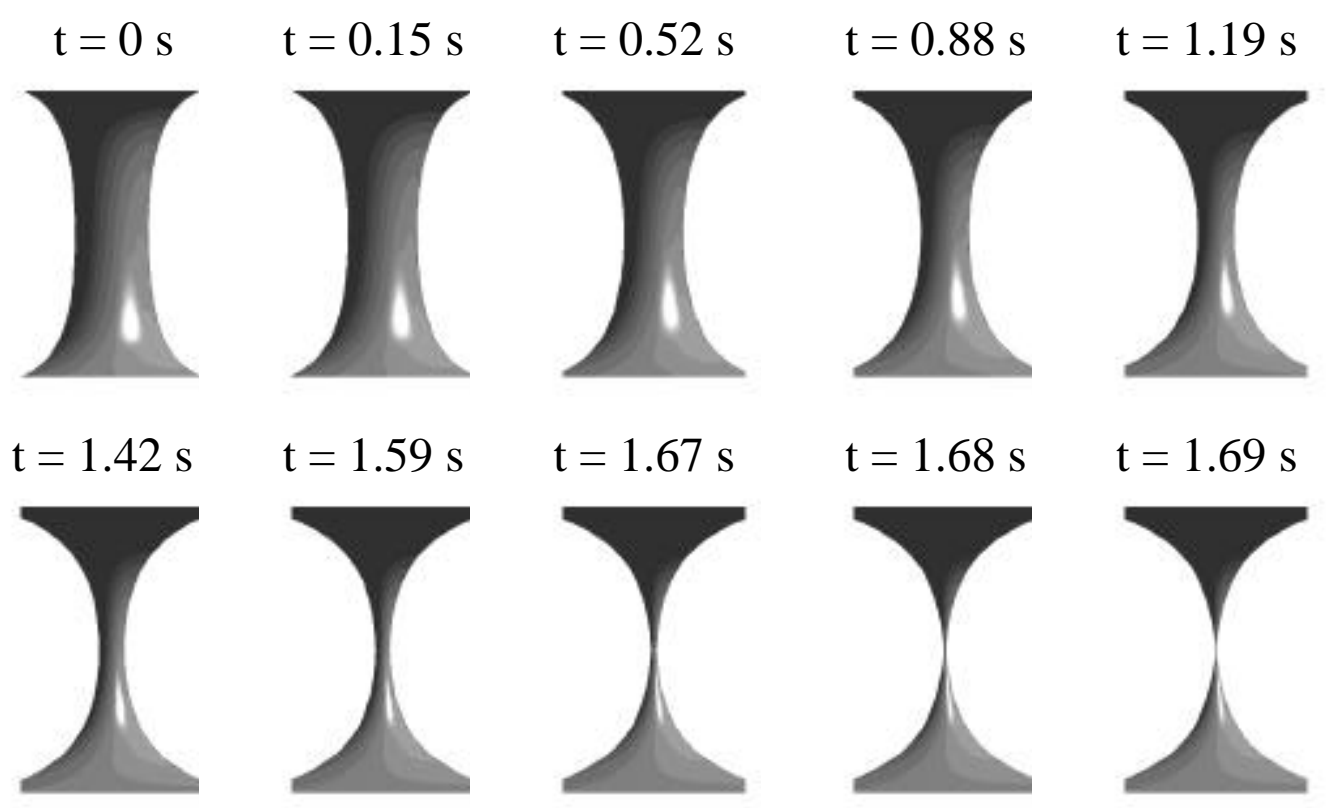

A5
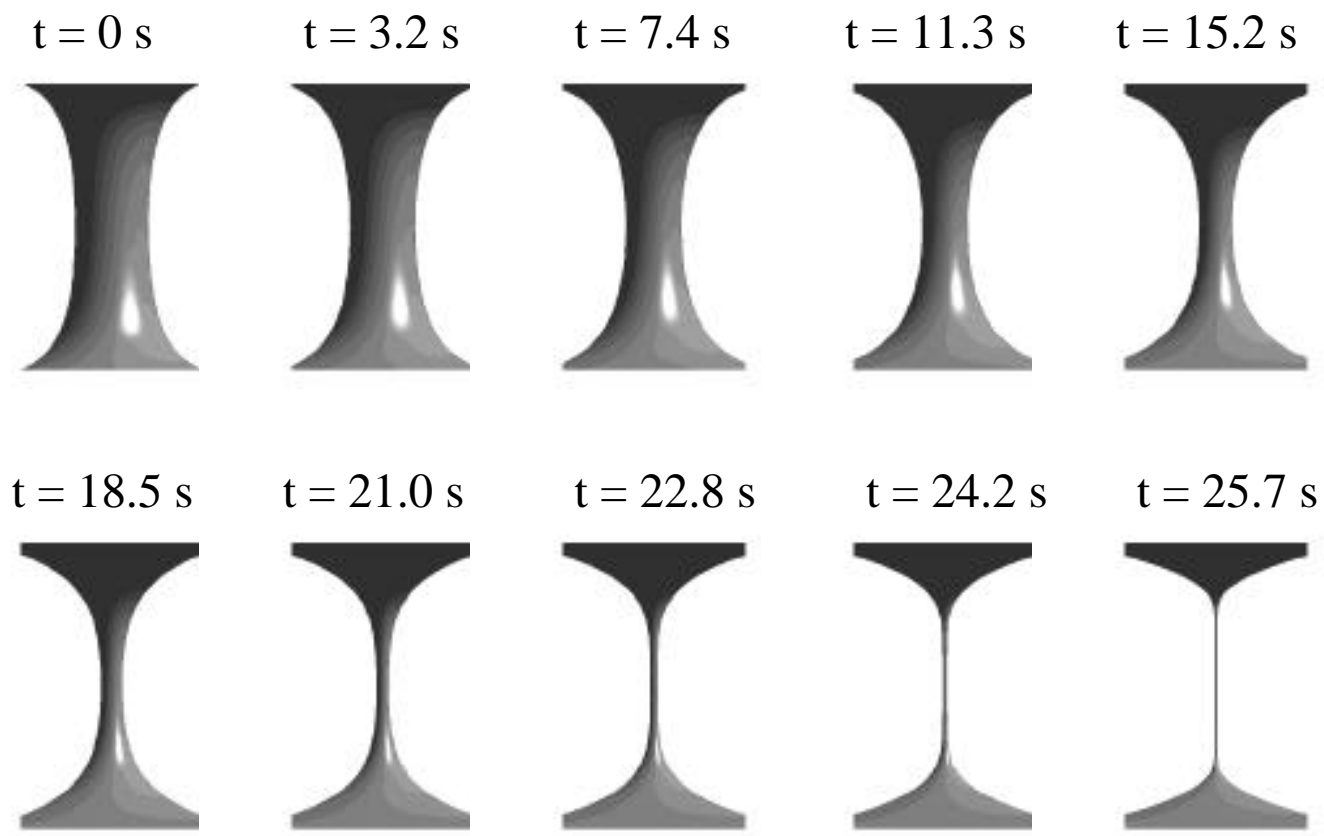

Figure 11 